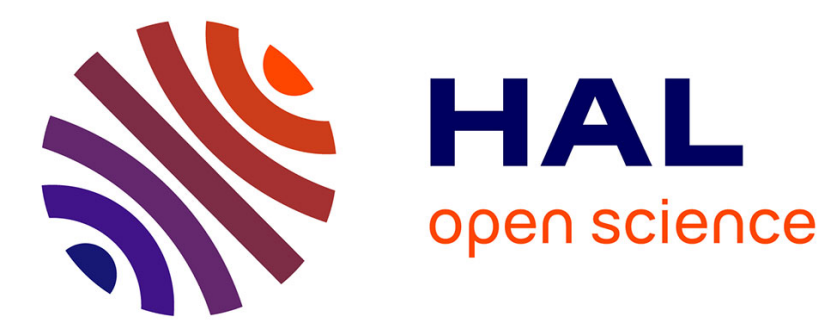

\title{
Time harmonic wave propagation in one dimensional weakly randomly perturbed periodic media \\ Sonia Fliss, Laure Giovangigli
}

\section{To cite this version:}

Sonia Fliss, Laure Giovangigli. Time harmonic wave propagation in one dimensional weakly randomly perturbed periodic media. SN Partial Differential Equations and Applications, In press, 1 (40). hal02504392

\section{HAL Id: hal-02504392 \\ https://hal.inria.fr/hal-02504392}

Submitted on 10 Mar 2020

HAL is a multi-disciplinary open access archive for the deposit and dissemination of scientific research documents, whether they are published or not. The documents may come from teaching and research institutions in France or abroad, or from public or private research centers.
L'archive ouverte pluridisciplinaire HAL, est destinée au dépôt et à la diffusion de documents scientifiques de niveau recherche, publiés ou non, émanant des établissements d'enseignement et de recherche français ou étrangers, des laboratoires publics ou privés. 
Noname manuscript No.

(will be inserted by the editor)

\title{
Time harmonic wave propagation in one dimensional weakly randomly perturbed periodic media
}

\author{
Sonia Fliss · Laure Giovangigli
}

Received: date / Accepted: date

\begin{abstract}
In this work we consider the solution of the time harmonic wave equation in a one dimensional periodic medium with weak random perturbations. More precisely, we study two types of weak perturbations: (1) the case of stationary, ergodic and oscillating coefficients, the typical size of the oscillations being small compared to the wavelength and (2) the case of rare random perturbations of the medium, where each period has a small probability to have its coefficients modified, independently of the other periods. Our goal is to derive an asymptotic approximation of the solution with respect to the small parameter. This can be used in order to construct absorbing boundary conditions for such media.
\end{abstract}

Keywords Wave equation $\cdot$ Random media $\cdot$ Periodic media

\section{Introduction and model problem}

The propagation of waves in periodic media has known a regain of interest for many important applications, particularly in optics for micro and nano-technology. However, in real applications, the media are often not perfectly periodic and the perturbations can be partially known. The use of randomness to model this partial knowledge is particularly well suited.

We want to propose a numerical method for computing the propagation of waves in such media. More precisely, we want to reduce the pure numerical computation to a bounded region, typically a region where the medium is well-known (i.e. not random). It is then necessary to construct transparent or absorbing boundary conditions to impose at the boundary of the computational domain. These conditions should reflect the best possible the wave propagation at the exterior medium. This paper is a first contribution to the construction of such boundary conditions in particular situations.

We are interested in this paper in the one-dimensional time harmonic wave equation in infinite media which are periodic with weak random perturbations. More precisely, let $(\Omega, \mathcal{F}, \mathbb{P})$ be a probability space. We consider a random medium occupying $\mathbb{R}$, characterised by the following coefficients

$$
\begin{array}{ll}
\text { for a.e. }(x, \omega) \in \mathbb{R} \times \Omega, & \kappa_{\varepsilon}(x, \omega)=\kappa_{\mathrm{per}}(x)+\tilde{\kappa}_{\varepsilon}(x, \omega), \\
& \rho_{\varepsilon}(x, \omega)=\rho_{\mathrm{per}}(x)+\tilde{\rho}_{\varepsilon}(x, \omega),
\end{array}
$$

where

\section{Sonia Fliss}

POEMS, Ensta Paris

828 boulevard des Maréchaux

91128 Palaiseau Cedex E-mail: sonia.fliss@ensta-paris.fr

Laure Giovangigli

POEMS, Ensta Paris

828 boulevard des Maréchaux

91128 Palaiseau Cedex E-mail: laure.giovangigli@ensta-paris.fr 
- $\kappa_{\varepsilon}$ and $\rho_{\varepsilon}$ are uniformly bounded from above and below, that is, that there exist strictly non negative constants $\mathcal{K}_{+}, \mathcal{K}_{-}, \rho_{+}, \rho_{-}$such that almost everywhere (a.e.) and almost surely (a.s.)

$$
0<\kappa_{-} \leq \kappa_{\varepsilon} \leq \kappa_{+}, \quad 0<\rho_{-} \leq \rho_{\varepsilon} \leq \rho_{+} .
$$

- $\kappa_{\text {per }}$ and $\rho_{\text {per }}$ are $L$-periodic functions with $L>0$ such that

$$
0<\kappa_{-} \leq \kappa_{\text {per }} \leq \kappa_{+}, \quad 0<\rho_{-} \leq \rho_{\text {per }} \leq \rho_{+}
$$

- $\tilde{\kappa}_{\varepsilon}$ and $\tilde{\rho}_{\varepsilon}$ are random coefficients, depending on a small parameter $\varepsilon$. We consider in this paper two types of weak random perturbations that are specified later. We suppose that there exists $a>0$ such that a.s.

$$
\operatorname{Supp} \tilde{\kappa}_{\varepsilon} \cap \operatorname{Supp} \tilde{\rho}_{\varepsilon} \subset \mathbb{R} \backslash[-a, a]
$$

We consider the time harmonic scalar wave equation (deduced from the wave equation assuming a time-dependence $e^{-\imath k t}$ at a given frequency $k$ ), also known as the Helmholtz equation, in this medium,

$$
-\partial_{x}\left[\kappa_{\varepsilon}(\cdot, \omega) \partial_{x} u_{\varepsilon}(\cdot, \omega)\right]-\rho_{\varepsilon}(\cdot, \omega) k^{2} u_{\varepsilon}(\cdot, \omega)=f \quad \text { in } \mathbb{R} \text {, for a.e. } \omega \in \Omega,
$$

where $f \in L^{2}(\mathbb{R})$ has a compact support. We can suppose without loss of generality that the support of $f$ is included in $(-a, a)$. Of course, if the coefficients are random, the solution, if any, will also be random.

It is well-known that the Helmholtz equation with real frequency is in general not well-posed in the classical $L^{2}$ setting. This is linked to the fact that the physical solution is in general not of finite energy (i.e. with a finite $L^{2}$ norm) since a propagation without attenuation is possible at infinity. On the other hand, in the $L_{\text {loc }}^{2}$ framework, an infinity of solutions can be found. Usually, radiation conditions which characterize the behaviour at infinity of the physical solution, have to be determined and added to the problem in order to recover a well-posed problem. When the medium is perfectly periodic or if it is a local perturbation of a periodic medium, the authors in [15,11] have derived radiation conditions and shown that the problem is well-posed. For random media and even for weakly random perturbations of periodic media, this question is still open. The classical framework to answer to this question is to use the limiting absorption principle. More precisely, we introduce a damping parameter $\eta$ and we define the physical solution of (2) as the limit (in a sense to be determined), as $\eta$ goes to 0 , of the unique $H^{1}$ solution of the Helmholtz equation with damping

$$
-\partial_{x}\left[\kappa_{\varepsilon}(\cdot, \omega) \partial_{x} u_{\varepsilon}^{\eta}(\cdot, \omega)\right]-\rho_{\varepsilon}(\cdot, \omega)\left(k^{2}+\imath \eta\right) u_{\varepsilon}^{\eta}(\cdot, \omega)=0 \text { in } \mathbb{R}, \text { for a.e. } \omega \in \Omega .
$$

The proof of the limiting absorption principle requires a good knowledge of the solution of the Helmholtz equation with damping. This is what we investigate here. The subject of the limiting absorption principle will be the subject of a forthcoming paper. In the whole paper, we fix the damping parameter, so we forget the subscript $\eta$. In other words, we consider (2) with $\operatorname{Im}\left(k^{2}\right)>0$ so that there exists a.s. a unique $H^{1}$ solution $u_{\varepsilon}$.

In order to restrict the computation in the bounded region $(-a, a)$, one would like to characterize the restriction of $u_{\varepsilon}$ to $(-a, a)$ by the solution of $(2)$ in $(-a, a)$ that satisfies boundary conditions of the form

$$
\pm\left[\kappa_{\varepsilon} \frac{\partial u_{\varepsilon}}{\partial x}\right](\cdot, \omega)+\lambda_{\varepsilon}^{ \pm}(\omega) u_{\varepsilon}(\cdot, \omega)=0 \text { on } \pm a, \text { for a.e. } \omega \in \Omega
$$

where $\lambda_{\varepsilon}^{ \pm}(\omega)$ are the Dirichlet-to-Neumann (DtN) coefficients defined by

$$
\lambda_{\varepsilon}^{ \pm}(\omega):=\mp\left[\kappa_{\varepsilon} \frac{\partial u_{\varepsilon}}{\partial x}\right]( \pm a, \omega) \text {, for a.e. } \omega \in \Omega
$$

and $u_{\varepsilon}^{ \pm}(\cdot, \omega)$ is the unique $H^{1}$ solution of the half-line problem

$$
\left\{\begin{array}{l}
-\partial_{x}\left[\kappa_{\varepsilon}(\cdot, \omega) \partial_{x} u_{\varepsilon}^{ \pm}(\cdot, \omega)\right]-\rho_{\mathcal{\varepsilon}}(\cdot, \omega) k^{2} u_{\varepsilon}^{ \pm}(\cdot, \omega)=0 \text { on }( \pm a, \pm \infty), \text { for a.e. } \omega \in \Omega \\
u_{\mathcal{\varepsilon}}^{ \pm}( \pm a, \omega)=1, \text { for a.e. } \omega \in \Omega .
\end{array}\right.
$$


For the periodic case, the construction of such DtN coefficients was proposed in [15]11]. A similar construction is not achievable in the present case, because of the partial knowledge of the exterior medium. Our goal here is to propose approximations of $\lambda_{\varepsilon}^{ \pm}(\omega)$ at different orders in $\varepsilon$ and quantify how these approximations approach the exact DtN coefficient. As you shall see, the zero-order approximation of $\lambda_{\varepsilon}^{ \pm}(\omega)$ is a DtN coefficient associated to a periodic medium. We want to understand if and in which sense the higher order terms could correct this first deterministic order term theoretically. Moreover, we do not want to only give a theoretical definition of these terms, we want also to be able to compute them and use them from a numerical point of view.

Finally, let us describe now the two types of weak random perturbations that we study in this paper.

1. the Homogenization regime. We suppose that for a.e. $x \in \mathbb{R}, \omega \in \Omega, \kappa_{\varepsilon}$ and $\rho_{\varepsilon}$ can be written as

$$
\kappa_{\varepsilon}(x)=\kappa_{\text {per }}(x)\left(1+v_{s}^{\kappa}\left(\frac{x}{\varepsilon}, \omega\right)\right)^{-1}, \quad \rho_{\varepsilon}(x, \omega)=\rho_{\text {per }}(x)\left(1+v_{s}^{\rho}\left(\frac{x}{\varepsilon}, \omega\right)\right),
$$

where $v_{s}^{\kappa}$ and $v_{s}^{\rho}$ are stationary and ergodic processes that are supposed to be with mean zero without loss of generality. When $\kappa_{p}$ and $\rho_{p}$ are constant (denoted resp. by $\kappa_{0}$ and $\rho_{0}$ ), this is the classical setting to apply stochastic homogenization theory (see for instance [18,22]). In that case, it is well known that when $\varepsilon$ goes to 0 , the family $\left(u_{\varepsilon}^{ \pm}\right)$converges almost surely weakly in $H^{1}( \pm a, \pm \infty)$ to the solution of the Helmholtz equation with effective constant coefficients $\kappa_{0}$ and $\rho_{0}$. Under additional assumptions on the coefficients (strongly mixing properties, short range of correlation,...) it is possible to provide error estimates in terms of $\varepsilon$. This is called the quantitative homogenization. This was investigated in lots of papers for any dimension (see for instance [14, [3]6]). A precise definition of the first corrector (i.e. the second term of the asymptotics in terms of $\varepsilon)$ was given in $1 \mathrm{D}$ in [5]13] provided that the coefficients satisfy some mixing coefficients. Under the same properties for $v_{s}^{\kappa}$ and $v_{s}^{\rho}$ (stationarity and strongly mixing property), we propose here an extension of these results when the coefficient $\kappa_{p}$ or $\rho_{p}$ are general periodic functions. Let us emphasize that to derive an asymptotic expansion of the DtN coefficient, we need a precise description on the flux $\kappa_{\varepsilon} \partial_{x} u_{\varepsilon}^{ \pm}$and not only the solution. See Section 3

2. Rare random perturbations case. We suppose here that for a.e. $x \in \mathbb{R}, \omega \in \Omega, \kappa_{\varepsilon}$ and $\rho_{\varepsilon}$ can be written as

$$
\begin{aligned}
& \tilde{\mathcal{K}}_{\varepsilon}(x, \omega)=b_{\mathcal{\varepsilon}}(x, \omega) \tilde{\kappa}_{\text {per }}(x), \\
& \tilde{\rho}_{\varepsilon}(x, \omega)=b_{\mathcal{\varepsilon}}(x, \omega) \tilde{\rho}_{\text {per }}(x),
\end{aligned}
$$

where $\tilde{\kappa}_{\text {per }}$ and $\tilde{\rho}_{\text {per }}$ are $L$-periodic functions and the random perturbation $b_{\varepsilon}$ is given by

$$
b_{\varepsilon}(x, \omega)=\sum_{j \in \mathbb{Z}} \mathbb{1}_{I_{j}}(x) B_{\varepsilon}^{j}(\omega), \quad I_{j}=[j L,(j+1) L] .
$$

Here $\left(B_{\varepsilon}^{j}\right)_{j \in \mathbb{N}}$ are independent Bernouilli random variables with parameter $\varepsilon \in[0,1]$. Intuitively, each period with parameters $\left\{\kappa_{\text {per }}, \rho_{\text {per }}\right\}$ is perturbed with probability $\varepsilon$, independently of the other periods, to have its coefficients modified to $\left\{\kappa_{\text {per }}+\tilde{\kappa}_{\text {per }}, \rho_{\text {per }}+\tilde{\rho}_{\text {per }}\right\}$. The idea of this problem originates from a paper [1] by C. Le Bris and A. Anantharaman who worked on this particular problem but in the context of homogenization to model composite materials with defects. [2] and [20] extend the framework to other random perturbations but are still considering the homogenized problem. We want here to investigate this case for the Helmholtz equation and in the general regime where homogenization techniques cannot necessarily be applied. See Section 4

\section{Preliminary results}

In the whole paper, for any Banach space $V$, we define

$$
L^{2}(\Omega, V):=\left\{u, \text { for a.e. } \omega \in \Omega, u(\cdot, \omega) \in V, \mathbb{E}\left[\|u(\cdot, \omega)\|_{V}^{2}\right]<+\infty\right\}
$$


equipped with the norm

$$
\forall u \in L^{2}(\Omega, V),\|u\|_{L^{2}(\Omega, V)}=\sqrt{\mathbb{E}\left[\|u(\cdot, \omega)\|_{V}^{2}\right]} .
$$

The approximations of the DtN coefficients $\lambda_{\varepsilon}^{ \pm}$at different orders in $\varepsilon$ are obtained from similar approximations of the half-line solutions $u_{\varepsilon}^{ \pm}$. The half-line problems (5) are similar but independent one from the other. We can then concentrate on one half-line problem to obtain approximations of the corresponding solution and the corresponding DtN coefficient. Without loss of generality, we suppose that $a=0$. In the sequel, we thus study the solution $u_{\varepsilon}^{+} \in L^{2}\left(\Omega, H^{1}\left(\mathbb{R}^{+}\right)\right)$of

$$
\left\{\begin{array}{l}
-\partial_{x}\left[\kappa_{\varepsilon} \partial_{x} u_{\varepsilon}^{+}\right]-\rho_{\varepsilon} k^{2} u_{\varepsilon}^{+}=0 \text { on } \mathbb{R}^{+}, \\
u_{\varepsilon}^{+}(0, \cdot)=1 .
\end{array}\right.
$$

and the corresponding DtN coefficient defined a.s. as

$$
\lambda_{\varepsilon}^{+}:=-\left[\kappa_{\varepsilon} \frac{\partial u_{\varepsilon}^{+}}{\partial x}\right](0, \cdot)
$$

The well-posedness of the half-line problem (7) is recalled in the following proposition.

Proposition 1 There exists a unique solution in $L^{2}\left(\Omega, H^{1}\left(\mathbb{R}^{+}\right)\right)$of (7).

Proof The proof is really classical for deterministic problem, we only extend it for our random setting. It is sufficient to show that for any $f \in L^{2}\left(\Omega, H^{-1}\left(\mathbb{R}^{+}\right)\right)$, there exists a unique solution in $L^{2}\left(\Omega, H_{0}^{1}\left(\mathbb{R}^{+}\right)\right)$of

$$
-\partial_{x}\left[\kappa_{\varepsilon} \partial_{x} v\right]-\rho_{\varepsilon} k^{2} v=f \text { on } \mathbb{R}^{+} .
$$

Let $a_{\varepsilon}: L^{2}\left(\Omega, H_{0}^{1}\left(\mathbb{R}_{+}^{*}\right)\right) \times L^{2}\left(\Omega, H_{0}^{1}\left(\mathbb{R}_{+}^{*}\right)\right) \rightarrow \mathbb{C}$ be the sesquilinear form defined by

$$
\forall v, w \in L^{2}\left(\Omega, H_{0}^{1}\left(\mathbb{R}_{+}^{*}\right)\right), \quad a_{\varepsilon}(v, w)=\mathbb{E}\left[\left\langle\kappa_{\varepsilon} \frac{\partial v}{\partial x}, \frac{\partial w}{\partial x}\right\rangle-k^{2}\left\langle\rho_{\varepsilon} v, w\right\rangle\right],
$$

where $\langle\cdot, \cdot\rangle$ denotes the scalar product in $L^{2}\left(\mathbb{R}_{+}^{*}\right)$. To apply Lax-Milgram theorem, the only difficulty comes from the proof of coercivity of $a_{\varepsilon}$. For all $v \in L^{2}\left(\Omega, H_{0}^{1}\left(\mathbb{R}_{+}^{*}\right)\right)$

$$
\begin{aligned}
\Im\left(\frac{1}{k} a(v, v)\right) & =\Im\left(\frac{1}{k} \mathbb{E}\left[\left\langle\kappa_{\eta} \frac{\partial v}{\partial x}, \frac{\partial v}{\partial x}\right\rangle-k^{2}\left\langle\rho_{\eta} v, v\right\rangle\right)\right] \\
& =-\Im(k) \mathbb{E}\left[\frac{1}{|k|^{2}}\left\langle\kappa_{\eta} \frac{\partial v}{\partial x}, \frac{\partial v}{\partial x}\right\rangle+\left\langle\rho_{\eta} v, v\right\rangle\right] .
\end{aligned}
$$

Thus, using that for all $z \in \mathbb{C},|z| \geq \Im z$, we obtain

$$
|a(v, v)| \geq \Im(k)|k| \min \left(\frac{\kappa_{-}}{|k|^{2}}, \rho_{-}\right)\|v\|_{L^{2}\left(\Omega, H^{1}\left(\mathbb{R}_{+}^{*}\right)\right)}^{2}
$$

Since we are working in presence of dissipation $\Im\left(k^{2}\right)>0$, we know that the solution's amplitude decreases exponentially to 0 . The first step in our study is to take advantage of this decaying property and approximate the solution $u_{\varepsilon}$ by the solution of the Helmholtz equation in a finite interval. So for any $N \in \mathbb{N}$, let us define $u_{\varepsilon}^{N}$ the unique solution in $L^{2}\left(\Omega, H^{1}((0, N L))\right)$ of the following problem

$$
\left\{\begin{array}{l}
-\partial_{x}\left[\kappa_{\varepsilon} \partial_{x} u_{\varepsilon}^{N}\right]-\rho_{\varepsilon} k^{2} u_{\varepsilon}^{N}=0 \text { on } \Omega \times(0, N L), \\
u_{\varepsilon}^{N}(0, \cdot)=1, \quad u_{\varepsilon}^{N}(N L, \cdot)=0
\end{array}\right.
$$

This problem is well-posed in $L^{2}\left(\Omega, H^{1}((0, N L))\right)$ as a direct consequence of the following proposition. 
Proposition 2 For any $f \in L^{2}\left(\Omega, H^{-1}(0, N L)\right)$, there exists a unique solution $v^{N}$ in $L^{2}\left(\Omega, H_{0}^{1}(0, N L)\right)$ of

$$
-\partial_{x}\left[\kappa_{\varepsilon} \partial_{x} v^{N}\right]-\rho_{\varepsilon} k^{2} v^{N}=\text { fon }(0, N L),
$$

and

$$
\left\|v^{N}\right\|_{L^{2}\left(\Omega, H^{1}((0, N L))\right)} \leq C\|f\|_{L^{2}\left(\Omega, H^{-1}((0, N L))\right)}
$$

where $C=\left[\Im(k)|k| \min \left(\frac{\kappa_{-}}{|k|^{2}}, \rho_{-}\right)\right]^{-1}$.

The proof is the same than the one of Proposition 1 .

Remark 1 The condition on $x=N L$ is a Dirichlet condition but it could be of course Neumann or any combination of the two.

We have the following estimate of the approximation of $u_{\varepsilon}$ by $u_{\varepsilon}^{N}$.

Proposition 3 There exists a constant $C>0$ depending on $k_{,} \kappa_{+}$and $\rho_{-}$such that for all $\alpha<\Im\left(k^{2}\right) \rho_{-} / \kappa_{+}$

$$
\left\|u_{\varepsilon}^{+}-u_{\varepsilon}^{N}\right\|_{L^{2}\left(\Omega, H^{1}((0, N L))\right)} \leq C e^{-\alpha N L} .
$$

We can then choose $N$ to obtain an error as small as we want to. Let us finally define the DtN coefficient associated to 9

$$
\lambda_{\varepsilon}^{N}:=-\left[\kappa_{\varepsilon} \frac{\partial u_{\varepsilon}^{N}}{\partial x}\right](0, \cdot)
$$

In the following sections, we propose now an asymptotic expansion of $u_{\varepsilon}^{N}$ and $\lambda_{\varepsilon}^{N}$ in terms of $\varepsilon$ for the two cases described at the end of the Introduction. In both cases, we expect that the limit behaviour of $u_{\varepsilon}^{N}$ or in other words, the first term in the asymptotic expansion, is the solution of the problem with the periodic coefficients $\left(\kappa_{\text {per }}, \rho_{\text {per }}\right)$. Let us define $u_{\mathrm{per}}^{N}$ the solution of the wave equation in the unperturbed domain $(0, N L) \cdot u_{\mathrm{per}}^{N}$ is the unique solution in $H^{1}((0, N L))$ of

$$
\left\{\begin{array}{l}
-\partial_{x}\left[\kappa_{\mathrm{per}} \partial_{x} u_{\mathrm{per}}^{N}\right]-k^{2} \rho_{\mathrm{per}} u_{\mathrm{per}}^{N}=0 \quad \text { in }(0, N L), \\
u_{\mathrm{per}}^{N}(0)=1, \quad u_{\mathrm{per}}^{N}(N L)=0 .
\end{array}\right.
$$

and $\lambda_{\text {per }}^{N}$ is the associated DtN coefficient defined by

$$
\lambda_{\text {per }}^{N}=-\left[\kappa_{\text {per }} \partial_{x} u_{\text {per }}^{N}\right](0) .
$$

\section{Homogenization regime}

We consider in this section the solution $u_{\varepsilon}^{N}$ of 9 ) when the coefficients $\kappa_{\varepsilon}$ and $\rho_{\varepsilon}$ are such that for a.e. $x \in \mathbb{R}^{+}$and $\omega \in \Omega$

$$
\kappa_{\varepsilon}(x)=\kappa_{p e r}(x)\left(1+v_{s}^{\kappa}\left(\frac{x}{\varepsilon}, \omega\right)\right)^{-1}, \quad \rho_{\varepsilon}(x, \omega)=\rho_{\text {per }}(x)\left(1+v_{s}^{\rho}\left(\frac{x}{\varepsilon}, \omega\right)\right),
$$

where we recall that $\kappa_{\text {per }}$ and $\rho_{\text {per }}$ are $L$-periodic functions and $v_{s}^{\kappa}$ and $v_{s}^{\rho}$ are jointly strictly stationary processes on $(\Omega, \mathcal{F}, \mathbb{P})$ that satisfy strongly mixing property. Without loss of generality, we suppose that $v_{s}^{\kappa}$ and $v_{s}^{\rho}$ have mean zero.

If $v_{s}^{\kappa}$ and $v_{s}^{\rho}$ are jointly strictly stationary ergodic processes and $\kappa_{\text {per }}$ and $\rho_{\text {per }}$ are constant (resp. $\kappa_{0}$ and $\rho_{0}$ ), we know from the stochastic homogenization theory that $u_{\varepsilon}^{N}$ converges weakly a.s. in $H^{1}((0, N L))$ to $u_{0}^{N}$ unique solution in $H^{1}((0, N L))$ of the following homogenized problem

$$
\left\{\begin{array}{l}
-\kappa_{0} d_{x}^{2} u_{0}^{N}-k^{2} \rho_{0} u_{0}^{N}=0 \quad \text { in }(0, N L), \\
u_{0}^{N}(0)=1, \quad u_{0}^{N}(N L)=0 .
\end{array}\right.
$$

We want to establish a similar result in the case of a perturbed periodic medium, quantify the rate of convergence and compute a corrector, i.e. the second term in the asymptotic expansion of $u_{\varepsilon}^{N}$ in 
terms of $\varepsilon$. As for the classical quantitative homogenization result, this is to quantify the rate of convergence of $u_{\varepsilon}^{N}$ and to exhibit a corrector that the mixing properties of $v_{s}^{\kappa}$ and $v_{s}^{\rho}$ are important. More precisely, we assume that $v_{s}^{\rho}$ and $v_{s}^{\kappa}$ verify jointly a specific type of strongly mixing assumption: they are jointly $\rho$-mixing processes with an integrable and decreasing coefficient $\varphi$. In the case of constant $\kappa_{\text {per }}$ and $\rho_{\text {per }}$, extensive work has been done to quantify the convergence of $u_{\varepsilon}^{N}$ to $u_{0}^{N}$ (see for example [10]). To extend the setting to periodic $\kappa_{\text {per }}$ and $\rho_{\text {per }}$ we chose to follow the specific approach of [5].

Before doing so, we recall elementary definitions and properties of the stationary and strongly mixing processes.

3.1 Stationary, ergodic and strongly mixing processes : definitions and properties

Definition 1 A process $f: \Omega \times \mathbb{R}^{p} \rightarrow \mathbb{R}^{p}$ is strictly stationary on $(\Omega, \mathcal{F}, \mathbb{P})$ if for all $n \in \mathbb{N}^{*}, x_{1}, \ldots x_{n} \in$ $\mathbb{R}^{p}, I_{1}, I_{2}, I_{n} \in \mathcal{B}\left(\mathbb{R}^{p}\right)$ and all $y \in \mathbb{R}$

$$
\mathbb{P}\left(f\left(x_{1}+y\right) \in I_{1}, \ldots f\left(x_{n}+y\right) \in I_{n}\right)=\mathbb{P}\left(f\left(x_{1}\right) \in I_{1}, \ldots f\left(x_{n}\right) \in I_{n}\right)
$$

In other words, the distribution of $f\left(x_{1}+y\right), f\left(x_{2}+y\right), \ldots, f\left(x_{n}+y\right)$ under $\mathbb{P}$ is independent of $y$.

Let $X$ denote the set of bounded measurable functions $g$ from $\left(\mathbb{R}^{p}, \mathcal{B}\left(\mathbb{R}^{p}\right)\right)$ to $\left(\mathbb{R}^{p}, \mathcal{B}\left(\mathbb{R}^{p}\right)\right)$ that verify a.e.

$$
\left|g_{i}\right| \leq C<\min \left(\rho_{-}, \rho_{+}, \kappa_{-}, \kappa_{+}\right), \forall i \in[|1, p|] .
$$

$\mathcal{B}\left(\mathbb{R}^{p}\right)$ denotes here the Borel $\sigma$-algebra of $\mathbb{R}^{p}$. We endow $X$ with the associated cylindrical $\sigma$-algebra $\mathcal{F}_{X}$, that is

$$
\mathcal{F}_{X}:=\sigma\left\{g \in X \mid g\left(x_{1}\right) \in A_{1}, \cdots, g\left(x_{n}\right) \in A_{n}\right\},
$$

where $n \in \mathbb{N}^{*}, x_{1}, \cdots, x_{n} \in \mathbb{R}^{p}, A_{1}, \cdots, A_{n} \in \mathcal{B}\left(\mathbb{R}^{p}\right)$.

For $y \in \mathbb{R}$, we introduce the shift operator on $X$ defined by

$$
\theta_{y} g=g(y+\cdot), \forall g \in X .
$$

If $f$ is a stationary process, we have that for all $y \in \mathbb{R}, A \in \mathcal{F}_{X}$

$$
\mathbb{P}_{f}(A)=\mathbb{P}_{f}\left(\theta_{y} A\right),
$$

where $\mathbb{P}_{f}$ is the law of $f$ defined by

$$
\mathbb{P}_{f}(A):=\mathbb{P}(f \in A) .
$$

We say that the shifts $\theta_{y}$ determine a measure-preserving flow in $\left(X, \mathcal{F}_{X}, \mathbb{P}_{f}\right)$.

Definition 2 A set $A \in \mathcal{F}_{X}$ is called invariant if for all $y \in \mathbb{R}$, we have $A=\theta_{y}^{-1} A$.

Definition 3 We say that the process $f$ is ergodic if for all $A \in \mathcal{F}_{X}$ invariant, $\mathbb{P}_{f}(A) \in\{0,1\}$.

We recall now the following fundamental result on stationary ergodic processes, on which is based the demonstration of the stochastic homogenization result for one dimensional problems. The proof of this result can be found in [24,19].

Theorem 1 (Ergodic theorem) We suppose that $f$ is a strictly stationary ergodic process. Let $g: X \rightarrow \mathbb{R}^{p}$ be a measurable function in $L^{1}\left(\mathbb{P}_{f}\right)$. $\mathbb{P}$-a.s. and in $L^{1}(\mathbb{P})$

$$
\frac{1}{2 T} \int_{-T}^{T} g(f(y+\cdot)) d y \underset{T \rightarrow+\infty}{\longrightarrow} \mathbb{E}[g(f)] .
$$

A sufficient condition for a stationary process to be ergodic is to be strongly mixing.

Definition 4 A stationary process $f$ is strongly mixing if for all $A, B \in \mathcal{F}_{X}$

$$
\mathbb{P}_{f}\left(A \cap \theta_{y}^{-1} B\right) \underset{y \rightarrow+\infty}{\longrightarrow} \mathbb{P}_{f}(A) \mathbb{P}_{f}(B) .
$$


Proposition 4 A stationary strongly mixing process is ergodic.

Proof Let $A$ be an invariant set of $\mathcal{F}_{\mathrm{X}}$. We take $B=A$ in the definition of the strongly mixing process. Since for all $y \in \mathbb{R}, \theta_{y}^{-1} A=A$ we obtain $\mathbb{P}_{f}(A)=\mathbb{P}_{f}(A)^{2}$. Thus $\mathbb{P}_{f}(A) \in\{0,1\}$.

Definition 5 The process $f$ is $\rho$-mixing with an integrable and decreasing coefficient $\varphi$ if there exists a function $\varphi \in L^{1}\left(\mathbb{R}_{+}^{*}\right)$ such that for all Borel sets $A, B \in \mathcal{B}(\mathbb{R})$ and all $f_{A} \in L^{2}\left(\Omega, \mathcal{F}_{A}, \mathbb{P}\right), f_{B} \in L^{2}\left(\Omega, \mathcal{F}_{B}, \mathbb{P}\right)$, the following inequality holds for all $i, j \in\{1, \ldots p\}$

$$
\left|\operatorname{Corr}\left(f_{A}, f_{B}\right)\right|=\left|\frac{\mathbb{E}\left[\left(f_{A}-\mathbb{E}\left[f_{A}\right]\right)\left(f_{B}-\mathbb{E}\left[f_{B}\right]\right)\right]}{\sqrt{\mathbb{E}\left[f_{A}^{2}\right] \mathbb{E}\left[f_{B}^{2}\right]}}\right| \leq \varphi(d(A, B)),
$$

where $\mathcal{F}_{A}$ (resp. $\mathcal{F}_{B}$ ) denotes the sub- $\sigma$-algebra of $\mathcal{F}$ generated by $f_{i}(x, \cdot)$ for $x \in A$ (resp. $f_{j}(x, \cdot)$ for $x \in B$ ). In this paper, we will also suppose that the corresponding function $\varphi$ is such that $\varphi^{1 / 2} \in L^{1}\left(\mathbb{R}_{+}^{*}\right)$.

Let us define the cross-correlation functions associated to a stationary process $f: \Omega \times \mathbb{R}^{p} \rightarrow \mathbb{R}^{p}$ defined by

$$
R_{f_{i}, f_{j}}(x):=\mathbb{E}\left[f_{i}(y) f_{j}(y+x)\right], \forall i, j_{\in}\{1, \ldots p\},
$$

where $f=\left(f_{1}, \ldots, f_{p}\right)$ and for all $i, f_{i}: \Omega \times \mathbb{R}^{p} \rightarrow \mathbb{R}$.

Note that since $f$ is strictly stationary, $\left(R_{f_{i}, f_{j}}\right)_{i, j}$ as defined before are independent on $y$. Note also that if $f$ is a strictly stationary and strongly mixing process then if $f$ is $\rho$-mixing, its cross correlation functions are integrable on $\mathbb{R}$ (take $A=\{y\}$ and $B=\{x+y\}$ ).

Finally we say that two processes $v^{\kappa}$ and $v^{\rho}$ are jointly strictly stationary and strongly mixing if $\left(v^{\kappa}, v^{\rho}\right)$ is strictly stationary and strongly mixing.

Let us now state two technical results that will be useful for our study.

Lemma 1 Let $a^{i}, i \in\{1, \ldots, 4\}$ be bounded zero-mean stationary jointly $\rho$-mixing processes with coefficient $\varphi \in L^{1}((0,+\infty))$ such that $\varphi^{1 / 2} \in L^{1}\left(\mathbb{R}_{+}^{*}\right)$. We suppose that $\mathbb{E}\left(\left|a^{i}\right|^{6}\right)<+\infty$. Let $a_{\varepsilon}^{i}:=a^{i}\left(\frac{\dot{v}}{\varepsilon}\right)$. For a fixed $D>0$, we have the following estimates

$$
\begin{gathered}
\int_{0}^{D} \int_{0}^{D}\left|\mathbb{E}\left[a_{\varepsilon}^{1}\left(t_{1}\right) a_{\varepsilon}^{2}\left(t_{2}\right)\right]\right| d t_{1} d t_{2} \leq C \varepsilon \\
\int_{0}^{D} \int_{0}^{D} \int_{0}^{D} \int_{0}^{D}\left|\mathbb{E}\left[a_{\varepsilon}^{1}\left(t_{1}\right) a_{\varepsilon}^{2}\left(t_{2}\right) a_{\varepsilon}^{3}\left(t_{3}\right) a_{\varepsilon}^{4}\left(t_{4}\right)\right]\right| d t_{1} d t_{2} d t_{3} d t_{4} \leq C \varepsilon^{2} \\
\int_{0}^{D} \int_{0}^{D} \int_{0}^{D} \int_{0}^{D}\left|\mathbb{E}\left[a_{\varepsilon}^{1}\left(t_{1}\right) a_{\varepsilon}^{2}\left(t_{2}\right) a_{\varepsilon}^{3}\left(t_{3}\right) a_{\varepsilon}^{4}\left(t_{4}\right)\right]\right|^{2} d t_{1} d t_{2} d t_{3} d t_{4} \leq C \varepsilon^{2} .
\end{gathered}
$$

Proof Let $0 \leq x_{1}, x_{2} \leq D$. By definition of the correlation function, we can express the left hand side of 15 as follows

$$
\int_{0}^{D} \int_{0}^{D}\left|\mathbb{E}\left[a_{\varepsilon}^{1}\left(t_{1}\right) a_{\varepsilon}^{2}\left(t_{2}\right)\right]\right| d t_{1} d t_{2}=\int_{0}^{D} \int_{0}^{D}\left|R_{a_{1}, a_{2}}\left(\frac{t_{1}-t_{2}}{\varepsilon}\right)\right| d t_{1} d t_{2}
$$

By a change of variables and integrability of $R_{a_{1}, a_{2}}$, we get

$$
\int_{0}^{D} \int_{0}^{D}\left|\mathbb{E}\left[a_{\varepsilon}^{1}\left(t_{1}\right) a_{\varepsilon}^{2}\left(t_{2}\right)\right]\right| d t_{1} d t_{2} \leq D \int_{\mathbb{R}}\left|R_{a_{1}, a_{2}}\left(\frac{x}{\varepsilon}\right)\right| d x \leq \varepsilon D\left\|R_{a_{1}, a_{2}}\right\|_{L^{1}(\mathbb{R})} .
$$


Let $\Sigma$ be the set of permutations of $\{1, \ldots, 4\}$. From [5, Lemma 2.1] (which gives us the first inequality) and thanks to a change of variables, we can show that

$$
\begin{aligned}
& \int_{0}^{D} \int_{0}^{D} \int_{0}^{D} \int_{0}^{D}\left|\mathbb{E}\left[a_{\varepsilon}^{1}\left(t_{1}\right) a_{\varepsilon}^{2}\left(t_{2}\right) a_{\epsilon}^{3}\left(t_{3}\right) a_{\epsilon}^{4}\left(t_{4}\right)\right]\right| d t_{1} d t_{2} d t_{3} d t_{4} \\
& \leq C \sum_{\sigma \in \Sigma} \int_{0}^{D} \int_{0}^{D} \int_{0}^{D} \int_{0}^{D} \varphi^{\frac{1}{2}}\left(\frac{\left|t_{\sigma(1)}-t_{\sigma(2)}\right|}{2 \varepsilon}\right) \varphi^{\frac{1}{2}}\left(\frac{\left|t_{\sigma(3)}-t_{\sigma(4)}\right|}{2 \varepsilon}\right) d t_{1} d t_{2} d t_{3} d t_{4} \\
& \leq 24 C D^{2} \int_{0}^{\infty} \int_{0}^{\infty} \varphi^{\frac{1}{2}}\left(\frac{\left|t_{1}\right|}{2 \varepsilon}\right) \varphi^{\frac{1}{2}}\left(\frac{\left|t_{2}\right|}{2 \varepsilon}\right) d t_{1} d t_{2} \\
& \leq 24 C \varepsilon^{2} D^{2}\left(\int_{0}^{\infty} \varphi^{\frac{1}{2}}(|s|) d s\right)^{2} .
\end{aligned}
$$

The inequality (17) is obtained in the exact same way except that $\varphi^{\frac{1}{2}}$ is replaced by $\varphi$ which is still integrable.

Lemma 2 Let $D>0, \mathcal{H}^{i}, i \in\{1,2\}$ be operators on $L^{2}(0, D)$ defined by

$$
\mathcal{H}^{i} f(x):=\int_{0}^{D} H^{i}(x, y) f(y) d y
$$

such that $H^{i}, i \in\{1,2\}$ are uniformly bounded functions on $\{0 \leq x, y \leq D\}$. Let $a^{i}, i \in\{1,2\}$ be bounded zero-mean stationary jointly $\rho$-mixing processes with coefficient $\varphi \in L^{1}(0,+\infty)$ such that $\varphi^{1 / 2} \in L^{1}\left(\mathbb{R}_{+}^{*}\right)$. We suppose that $\mathbb{E}\left(\left|a^{i}\right|^{6}\right)<+\infty$. Let $a_{\varepsilon}^{i}:=a^{i}\left(\frac{\dot{ }}{\varepsilon}\right)$. Then there exists a positive constant $C$ such that

$$
\begin{gathered}
\forall f \in L^{2}(0, D), \quad \mathbb{E}\left[\left\|\mathcal{H}^{1}\left[a_{\varepsilon}^{1} \mathcal{H}^{2}\left[a_{\varepsilon}^{2} f\right]\right]\right\|^{2}\right] \leq C \varepsilon\|f\|_{L^{2}}^{2} \\
\forall f \in L^{\infty}(0, D), \quad \mathbb{E}\left[\left\|\mathcal{H}^{1}\left[a_{\varepsilon}^{1} f\right]\right\|^{2}\right] \leq C \varepsilon\|f\|_{L^{\infty}}^{2}, \quad \mathbb{E}\left[\left\|\mathcal{H}^{1}\left[a_{\varepsilon}^{1} \mathcal{H}^{2}\left[a_{\varepsilon}^{2} f\right]\right]\right\|^{2}\right] \leq C \varepsilon^{2}\|f\|_{L^{\infty}}^{2} .
\end{gathered}
$$

Proof Let $f \in L^{2}(0, D)$,since $H^{i}$ are uniformly bounded on $(0, D)$ we obtain

$$
\begin{aligned}
\mathbb{E}\left[\left\|\mathcal{H}^{1}\left[a_{\varepsilon}^{1} \mathcal{H}^{2}\left[a_{\varepsilon}^{2} f\right]\right]\right\|^{2}\right] & =\mathbb{E}\left[\int_{0}^{D}\left|\int_{0}^{D} H^{1}(x, y) a_{\varepsilon}^{1}(y) \int_{0}^{D} H^{2}(y, z) a_{\varepsilon}^{2}(z) f(z) d z d y\right|^{2} d x\right] \\
& \leq\|f\|_{L^{2}}^{2} \mathbb{E}\left[\int_{0}^{D} \int_{0}^{D}\left|\int_{0}^{D} H^{1}(x, y) H^{2}(y, z) a_{\varepsilon}^{1}(y) a_{\varepsilon}^{2}(z) d y\right|^{2} d z d x\right] \\
& \leq\|f\|_{L^{2}}^{2} \mathbb{E}\left[\int_{0}^{D} \int_{0}^{D} \int_{0}^{D} \int_{0}^{D} H^{1}(x, y) H^{2}(y, z) \bar{H}^{1}\left(x, y^{\prime}\right) \bar{H}^{2}\left(y^{\prime}, z\right)\right. \\
& \leq C\|f\|_{L^{2}}^{2} \int_{0}^{D} \int_{0}^{D} \int_{0}^{D} \mid \mathbb{E}\left[\left(a_{\varepsilon}^{2}(z)\right)^{2} a_{\varepsilon}^{1}(y) a_{\varepsilon}^{1}\left(y^{\prime}\right) d y d y^{\prime} d z d x\right]
\end{aligned}
$$

Following the proof of Lemma (1), we use Lemma (3.1) from [5] and get

$$
\begin{aligned}
\left|\mathbb{E}\left[a_{\varepsilon}^{2}(z)^{2} a_{\varepsilon}^{1}(y) a_{\varepsilon}^{1}\left(y^{\prime}\right)\right]\right| \leq \varphi^{\frac{1}{2}}(0) \varphi^{\frac{1}{2}}\left(\frac{\left|y-y^{\prime}\right|}{2 \varepsilon}\right) & +\varphi^{\frac{1}{2}}\left(\frac{|z-y|}{2 \varepsilon}\right) \varphi^{\frac{1}{2}}\left(\frac{\left|y-y^{\prime}\right|}{2 \varepsilon}\right) \\
& +\varphi^{\frac{1}{2}}\left(\frac{\left|z-y^{\prime}\right|}{2 \varepsilon}\right) \varphi^{\frac{1}{2}}\left(\frac{\left|y-y^{\prime}\right|}{2 \varepsilon}\right)
\end{aligned}
$$

We thus obtain after changing variable and using the integrability of $\varphi^{1 / 2}$

$$
\mathbb{E}\left[\left\|\mathcal{H}^{1}\left[a_{\varepsilon}^{1} \mathcal{H}^{2}\left[a_{\varepsilon}^{2} f\right]\right]\right\|^{2}\right] \leq \varepsilon C\|f\|^{2}
$$


We have moreover for all $f \in L^{\infty}([0, D])$

$$
\begin{aligned}
\mathbb{E}\left[\left\|\mathcal{H}^{1}\left[a_{\varepsilon}^{1} f\right]\right\|^{2}\right] & =\mathbb{E}\left[\int_{0}^{D}\left|\int_{0}^{D} H^{1}(x, y) a_{\varepsilon}^{1}(y) f(y) d y\right|^{2} d x\right], \\
& =\int_{0}^{D} \int_{0}^{D} \int_{0}^{D} H^{1}(x, t) \bar{H}^{1}\left(x, t^{\prime}\right) \mathbb{E}\left[a_{\varepsilon}^{1}(t) a_{\varepsilon}^{1}\left(t^{\prime}\right)\right] f(t) \bar{f}\left(t^{\prime}\right) d t d t^{\prime} d x, \\
& \leq C\|f\|_{\infty}^{2} \int_{0}^{D} \int_{0}^{D} \int_{0}^{D}\left|\mathbb{E}\left[a_{\varepsilon}^{1}(t) a_{\varepsilon}^{1}\left(t^{\prime}\right)\right]\right| d t d t^{\prime} d x .
\end{aligned}
$$

The first inequality (15) in Lemma (1) allows finally to show that

$$
\mathbb{E}\left[\left\|\mathcal{H}^{1}\left[a_{\varepsilon}^{1} f\right]\right\|^{2}\right] \leq \varepsilon C\|f\|_{\infty}^{2} .
$$

We proceed in the same manner to obtain the last estimate. Indeed, for all $f \in L^{\infty}([0, D])$

$$
\begin{aligned}
\mathbb{E}\left[\left\|\mathcal{H}^{1}\left[a_{\varepsilon}^{1} \mathcal{H}^{2}\left[a_{\varepsilon}^{2} f\right]\right]\right\|^{2}\right] & =\mathbb{E}\left[\int_{0}^{D}\left|\int_{0}^{D} H^{1}(x, y) a_{\varepsilon}^{1}(y) \int_{0}^{D} H^{2}(y, z) a_{\varepsilon}^{2}(z) f(z) d z d y\right|^{2} d x\right], \\
& =\int_{0}^{D} \int_{0}^{D} \int_{0}^{D} \int_{0}^{D} \int_{0}^{D} H^{1}(x, y) \bar{H}^{1}\left(x, y^{\prime}\right) H^{2}(y, z) \bar{H}^{2}\left(y^{\prime}, z^{\prime}\right) \\
& \mathbb{E}\left[a_{\varepsilon}^{1}(y) a_{\varepsilon}^{1}\left(y^{\prime}\right) a_{\varepsilon}^{2}(z) a_{\varepsilon}^{2}\left(z^{\prime}\right)\right] f(z) \bar{f}\left(z^{\prime}\right) d y d y^{\prime} d z d z^{\prime} d x, \\
& \leq C\|f\|_{\infty}^{2} \int_{0}^{D} \int_{0}^{D} \int_{0}^{D} \int_{0}^{D}\left|\mathbb{E}\left[a_{\varepsilon}^{1}(y) a_{\varepsilon}^{1}\left(y^{\prime}\right) a_{\varepsilon}^{2}(z) a_{\varepsilon}^{2}\left(z^{\prime}\right)\right]\right| d y d y^{\prime} d z d z^{\prime} .
\end{aligned}
$$

The second inequality (15) in Lemma (1) gives us

$$
\mathbb{E}\left[\left\|\mathcal{H}^{1}\left[a_{\varepsilon}^{1} \mathcal{H}^{2}\left[a_{\varepsilon}^{2} f\right]\right]\right\|^{2}\right] \leq \varepsilon^{2} C\|f\|_{\infty}^{2} .
$$

In this section, we suppose that $v_{s}^{\kappa}$ and $v_{s}^{\rho}$ are zero-mean stationary jointly $\rho$-mixing processes with coefficient $\varphi \in L^{1}((0,+\infty))$ such that $\varphi^{1 / 2} \in L^{1}(0,+\infty)$.

\subsection{The limit behaviour}

We expect that the solution $u_{\varepsilon}^{N}$ of $(9)$ tends, in a certain sense to be determined, to the solution $u_{\mathrm{per}}^{N}$ of the problem (41) with the periodic coefficients $\left(\kappa_{\text {per }}, \rho_{\text {per }}\right)$.

Let us introduce the Green function $G$ associated to the periodic problem as follows: for all $y \in$ $(0, N L), G(\cdot, y)$ is the unique solution in $H^{1}(0, N L)$ of

$$
\left\{\begin{array}{l}
-\partial_{x}\left[\kappa_{\text {per }} \partial_{x} G(\cdot, y)\right]-k^{2} \rho_{\text {per }} G(\cdot, y)=\delta(\cdot-y) \quad \text { in }(0, N L), \\
G(0, y)=0, \quad G(N L, y)=0
\end{array}\right.
$$

$u_{\text {per }}^{N}$ and $u_{\varepsilon}^{N}$ can then be expressed for all $y \in[0, N L]$ as

$$
\begin{gathered}
u_{\text {per }}^{N}(y)=\kappa_{\text {per }}(0) \partial_{x} G(0, y), \\
u_{\varepsilon}^{N}(y)=-\int_{0}^{N L} \partial_{x} G(x, y)\left[\tilde{\kappa}_{\varepsilon} \partial_{x} u_{\varepsilon}^{N}\right](x) d x+k^{2} \int_{0}^{N L} G(x, y)\left[\tilde{\rho}_{\varepsilon} u_{\varepsilon}^{N}\right](x) d x+u_{\text {per }}^{N}(y),
\end{gathered}
$$

where we recall that $\tilde{\kappa}_{\varepsilon}:=\kappa_{\varepsilon}-\kappa_{\text {per }}$ and $\tilde{\rho}_{\varepsilon}:=\rho_{\varepsilon}-\rho_{\text {per }}$.

Let us introduce the volume integral operators $\mathcal{G}$ and $\mathcal{G}_{1}$ on $L^{2}(0, N L)$ defined by

$$
\forall f \in L^{2}(0, N L), \forall y \in(0, N L), \mathcal{G} f(y)=\int_{0}^{N L} G(x, y) f(x) d x \text { and } \mathcal{G}_{1} f(y)=\int_{0}^{N L} \partial_{x} G(x, y) f(x) d x,
$$

$u_{\varepsilon}^{N}$ reads then

$$
u_{\varepsilon}^{N}=-\mathcal{G}_{1}\left[\frac{\tilde{\kappa}_{\varepsilon}}{\mathcal{K}_{\varepsilon}} v_{\varepsilon}^{N}\right]+k^{2} \mathcal{G}\left[\tilde{\rho}_{\varepsilon} u_{\varepsilon}^{N}\right]+u_{\text {per }}^{N}
$$


where we have introduced $v_{\varepsilon}^{N}:=\kappa_{\varepsilon} \partial_{x} u_{\varepsilon}^{N}$. Since $u_{\varepsilon}^{N}$ is solution of (9), it is easy to see that $v_{\varepsilon}^{N}$, corresponding to a flux quantity, is solution of the following equation

$$
\left\{\begin{array}{l}
-\partial_{x}\left[\frac{1}{\rho_{\varepsilon}} \partial_{x} v_{\varepsilon}^{N}\right]-\frac{k^{2}}{\kappa_{\varepsilon}} v_{\varepsilon}^{N}=0 \quad \text { in }(0, N L), \\
-\left[\frac{1}{\rho_{\varepsilon}} \partial_{x} v_{\varepsilon}^{N}\right](0)=k^{2} \text { and }\left[\frac{1}{\rho_{\varepsilon}} \partial_{x} v_{\varepsilon}^{N}\right](N L)=0 .
\end{array}\right.
$$

We expect that $v_{\varepsilon}^{N}$ tends to $v_{\text {per }}^{N}:=\kappa_{\text {per }} \partial_{x} u_{\text {per }}^{N}$, where $v_{\text {per }}^{N}$ is solution of the same problem as $v_{\varepsilon}^{N}$ but replacing $\kappa_{\varepsilon}$ (resp. $\rho_{\varepsilon}$ ) by $\kappa_{\text {per }}$ (resp. $\rho_{\text {per }}$ ). The associated Green function, denoted by $G^{*}$, is defined as follows: for all $y \in(0, N L), G^{*}(\cdot, y)$ is the unique solution in $H^{1}(0, N L)$ of

$$
\left\{\begin{array}{l}
-\partial_{x}\left[\frac{1}{\rho_{\mathrm{per}}} \partial_{x} G^{*}(\cdot, y)\right]-\frac{k^{2}}{\kappa_{\mathrm{per}}} G^{*}(\cdot, y)=\delta(\cdot-y) \quad \text { in }(0, N L), \\
{\left[\frac{1}{\rho_{\mathrm{per}}} \partial_{x} G^{*}\right](0, y)=0 \text { and }\left[\frac{1}{\rho_{\mathrm{per}}} \partial_{x} G^{*}\right](N L, y)=0 .}
\end{array}\right.
$$

As previously, $v_{\mathrm{per}}^{N}$ and $v_{\varepsilon}^{N}$ can then be written for all $y \in(0, N L)$ as

$$
\begin{gathered}
v_{\mathrm{per}}^{N}(y)=k^{2} G(0, y), \\
v_{\varepsilon}^{N}(y)=\int_{0}^{N L} \partial_{x} G^{*}(x, y)\left[\frac{\tilde{\rho}_{\varepsilon}}{\rho_{\text {per }} \rho_{\varepsilon}} \partial_{x} v_{\varepsilon}^{N}\right](x) d x-k^{2} \int_{0}^{N L} G^{*}(x, y)\left[\frac{\tilde{\mathcal{K}}_{\varepsilon}}{\kappa_{\text {per }} \kappa_{\varepsilon}} v_{\varepsilon}^{N}\right](x) d x+v_{\mathrm{per}}^{N}(y),
\end{gathered}
$$

By introducing the associated volume integral operators $\mathcal{G}^{*}$ and $\mathcal{G}_{1}^{*}$ defined by

$\forall f \in L^{2}(0, N L), \forall y \in(0, N L), \mathcal{G}^{*} f(y)=\int_{0}^{N L} G^{*}(x, y) f(x) d x$ and $\mathcal{G}_{1}^{*} f(y)=\int_{0}^{N L} \partial_{x} G^{*}(x, y) f(x) d x$, $v_{\varepsilon}^{N}$ reads then

$$
v_{\varepsilon}^{N}=-k^{2} \mathcal{G}_{1}^{*}\left[\frac{\tilde{\rho}_{\varepsilon}}{\rho_{\text {per }}} u_{\varepsilon}^{N}\right]-k^{2} \mathcal{G}^{*}\left[\frac{\tilde{\kappa}_{\varepsilon}}{\kappa_{p e r} \kappa_{\varepsilon}} v_{\varepsilon}^{N}\right]+v_{\mathrm{per}}^{N}
$$

where we have used that $u_{\varepsilon}^{N}=-\frac{1}{k^{2} \rho_{\varepsilon}} d_{x} v_{\varepsilon}^{N}$.

Thanks to the two expressions (21) and (20), we can show the expected convergence result with a quantification of the convergence. The result is the same than the classical one of the stochastic homogenization theory, obtained in [5] and [13].

Theorem 2 There exists a constant $C$ such that

$$
\begin{gathered}
\left\|u_{\varepsilon}^{N}-u_{p e r}^{N}\right\|_{L^{2}\left(\Omega, L^{2}((0, N L))\right)} \leq C \sqrt{\varepsilon},\left\|v_{\varepsilon}^{N}-v_{p e r}^{N}\right\|_{L^{2}\left(\Omega, L^{2}((0, N L))\right)} \leq C \sqrt{\varepsilon} \\
\text { and }\left\|\lambda_{\varepsilon}^{N}-\lambda_{p e r}^{N}\right\|_{L^{2}(\Omega)} \leq C \sqrt{\varepsilon} .
\end{gathered}
$$

Proof First note that $G, \partial_{x} G, G^{*}$ and $\partial_{x} G^{*}$ are uniformly bounded in $\{0 \leq x, y \leq N L\}$. Moreover, for a.e. $x \in(0, N L)$,

$$
\frac{\tilde{\mathcal{K}}_{\varepsilon}(x, \cdot)}{\mathcal{K}_{\varepsilon}(x, \cdot)}=-v_{s}^{\kappa}\left(\frac{x}{\varepsilon}, \cdot\right) \text { and } \frac{\tilde{\rho}_{\varepsilon}(x, \cdot)}{\rho_{\text {per }}(x)}=v_{s}^{\rho}\left(\frac{x}{\varepsilon}, \cdot\right)
$$

We have assumed that $v_{s}^{\kappa}$ and $v_{s}^{\rho}$ are zero-mean stationary jointly $\rho$-mixing processes with coefficient $\varphi \in L^{1}((0,+\infty))$ such that $\varphi^{1 / 2} \in L^{1}(0,+\infty)$. From the proof of proposition 2, we know that $u_{\varepsilon}^{N}$ and $v_{\varepsilon}^{N}$ are bounded in $H^{1}(0, N L)$ uniformly in $\omega$ and $\varepsilon$. The continuous embedding of $H^{1}(0, N L)$ in $L^{\infty}(0, N L)$ implies that $u_{\varepsilon}^{N}$ and $v_{\varepsilon}^{N}$ are bounded in $L^{\infty}(0, N L)$ uniformly in $\omega$ and $\varepsilon$. Moreover, $\kappa_{\text {per }}$ and $\rho_{\text {per }}$ are bounded from below by a strictly positive constant.

We can therefore apply the estimate 19 of Lemma (2) and get the existence of a constant $C>0$ such that

$$
\mathbb{E}\left[\left\|\mathcal{G}_{1}\left[\frac{\tilde{\kappa}_{\varepsilon}}{\mathcal{K}_{\varepsilon}} v_{\varepsilon}^{N}\right]\right\|^{2}\right]+\mathbb{E}\left[\left\|k^{2} \mathcal{G}\left[\tilde{\rho}_{\varepsilon} u_{\varepsilon}^{N}\right]\right\|^{2}\right] \leq C \varepsilon
$$


This allow us to get the estimate on $\mathbb{E}\left[\left\|u_{\varepsilon}^{N}-u_{0}^{N}\right\|^{2}\right]$. Similar arguments hold to estimate $\mathbb{E}\left[\| v_{\varepsilon}^{N}-\right.$ $\left.v_{0}^{N} \|^{2}\right]$.

Finally, by definition (11) and (13), $\lambda_{\varepsilon}^{N}$ and $\lambda_{\text {per }}^{N}$ are nothing else but

$$
\lambda_{\varepsilon}^{N}=v_{\varepsilon}^{N}(0) \quad \text { and } \lambda_{\mathrm{per}}^{N}=v_{\mathrm{per}}^{N}(0) .
$$

Then using (21), we have

$$
\lambda_{\varepsilon}^{N}-\lambda_{\text {per }}^{N}=-k^{2} \int_{0}^{N L} \partial_{x} G^{*}(x, 0)\left[\frac{\tilde{\rho}_{\varepsilon}}{\rho_{\text {per }}} u_{\varepsilon}^{N}\right](x) d x-k^{2} \int_{0}^{N L} G^{*}(x, 0)\left[\frac{\tilde{\kappa}_{\varepsilon}}{\kappa_{\text {per }} \kappa_{\varepsilon}} v_{\varepsilon}^{N}\right](x) d x .
$$

By using that $G^{*}(\cdot, 0), G^{*}(\cdot, 0), u_{\varepsilon}^{N}$ and $v_{\varepsilon}^{N}$ are uniformly bounded in $(0, N L)$ and that $\kappa_{\text {per }}$ is bounded from below by a strictly positive constant, we show easily that there exists a constant $C>0$ such that

$$
\mathbb{E}\left[\left|\lambda_{\varepsilon}^{N}-\lambda_{\text {per }}^{N}\right|^{2}\right] \leq C \mathbb{E}\left[\int_{0}^{N L} \int_{0}^{N L} v_{s}^{\rho}\left(\frac{x}{\varepsilon}\right) v_{s}^{\rho}\left(\frac{y}{\varepsilon}\right) d x d y+\int_{0}^{N L} \int_{0}^{N L} v_{s}^{\kappa}\left(\frac{x}{\varepsilon}\right) v_{s}^{\kappa}\left(\frac{y}{\varepsilon}\right) d x d y\right] .
$$

It is sufficient to use the estimate (15) of Lemma 1 to conclude.

\subsection{Study of the first order corrector}

We need now to identify the term of order $\sqrt{\varepsilon}$ in the error terms. By looking at (20) and 21), since $u_{\varepsilon}^{N} \approx u_{\mathrm{per}}^{N}$ and $v_{\varepsilon}^{N} \approx v_{\mathrm{per}}^{N}$, a natural intuition is that

$$
u_{\varepsilon}^{N}-u_{\mathrm{per}}^{N} \approx-\mathcal{G}_{1}\left[\frac{\tilde{\kappa}_{\varepsilon}}{\mathcal{K}_{\varepsilon}} v_{\mathrm{per}}^{N}\right]+k^{2} \mathcal{G}\left[\tilde{\rho}_{\varepsilon} u_{\mathrm{per}}^{N}\right] \text { and } v_{\varepsilon}^{N}-v_{\mathrm{per}}^{N} \approx-k^{2} \mathcal{G}_{1}^{*}\left[\frac{\tilde{\rho}_{\varepsilon}}{\rho_{\text {per }}} u_{\mathrm{per}}^{N}\right]-k^{2} \mathcal{G}^{*}\left[\frac{\tilde{\kappa}_{\varepsilon}}{\kappa_{\text {per }} \mathcal{K}_{\varepsilon}} v_{\mathrm{per}}^{N}\right]
$$

We prove and quantify this approximation in the following proposition.

Proposition 5 The following estimate holds

$$
\begin{gathered}
\left\|u_{\varepsilon}^{N}-u_{p e r}^{N}-\left(-\mathcal{G}_{1}\left[\frac{\tilde{\mathcal{K}}_{\varepsilon}}{\mathcal{\kappa}_{\varepsilon}} v_{p e r}^{N}\right]+k^{2} \mathcal{G}\left[\tilde{\rho}_{\varepsilon} u_{p e r}^{N}\right]\right)\right\|_{L^{2}\left(\Omega, L^{2}(0, N L)\right)} \leq C \varepsilon, \\
\left\|v_{\varepsilon}^{N}-v_{p e r}^{N}-\left(-k^{2} \mathcal{G}_{1}^{*}\left[\frac{\tilde{\rho}_{\varepsilon}}{\rho_{p e r}} u_{p e r}^{N}\right]-k^{2} \mathcal{G}^{*}\left[\frac{\tilde{\kappa}_{\varepsilon}}{\kappa_{p e r} \kappa_{\varepsilon}} v_{p e r}^{N}\right]\right)\right\|_{L^{2}\left(\Omega, L^{2}(0, N L)\right)} \leq C \varepsilon .
\end{gathered}
$$

Proof Let us denote

$$
U_{\varepsilon}=u_{\varepsilon}^{N}-u_{\mathrm{per}}^{N}-\left(-\mathcal{G}_{1}\left[\frac{\tilde{\kappa}_{\varepsilon}}{\kappa_{\varepsilon}} v_{\text {per }}^{N}\right]+k^{2} \mathcal{G}\left[\tilde{\rho}_{\varepsilon} u_{\text {per }}^{N}\right]\right)
$$

Using (20), we can show that

$$
U_{\varepsilon}=\mathcal{G}_{1}\left[\frac{\tilde{\kappa}_{\varepsilon}}{\mathcal{K}_{\varepsilon}}\left(v_{\varepsilon}^{N}-v_{\mathrm{per}}^{N}\right)\right]-k^{2} \mathcal{G}\left[\tilde{\rho}_{\varepsilon}\left(u_{\varepsilon}^{N}-u_{\mathrm{per}}^{N}\right)\right] .
$$

We know that $u_{\varepsilon}^{N}, u_{\mathrm{per}}^{N}, v_{\varepsilon}^{N}$ and $v_{\mathrm{per}}^{N}$ are in $L^{\infty}(0, N L)$, so one could use the first part of [19]. However, we would have in that case only $\left\|U_{\varepsilon}\right\|_{L^{2}\left(\Omega, L^{2}(0, N L)\right)} \sim \sqrt{\varepsilon}$. Instead, we should use that $\left\|u_{\varepsilon}^{N}-u_{\text {per }}^{N}\right\|$ and $\left\|v_{\varepsilon}^{N}-v_{\text {per }}^{N}\right\|$ are small in $L^{2}\left(\Omega, L^{2}(0, N L)\right)$ and of order $\sqrt{\varepsilon}$ in order to conclude. In order to use the estimate (18), we have to use the same trick as in [5] and replace in the expression $u_{\varepsilon}^{N}$ of [20], the expression (21) of $v_{\varepsilon}^{N}$ and reuse the expression (20) of $u_{\varepsilon}^{N}$. We obtain

$$
\begin{aligned}
u_{\varepsilon}^{N}=u_{\mathrm{per}}^{N}-\mathcal{G}_{1}\left[\frac{\tilde{\mathcal{K}}_{\varepsilon}}{\mathcal{K}_{\varepsilon}} v_{\mathrm{per}}^{N}\right]+k^{2} \mathcal{G}\left[\tilde{\rho}_{\varepsilon} u_{\mathrm{per}}^{N}\right]+k^{2} \mathcal{G}_{1}\left[\frac{\tilde{\mathcal{K}}_{\varepsilon}}{\mathcal{K}_{\varepsilon}} \mathcal{G}_{1}^{*}\left[\frac{\tilde{\rho}_{\varepsilon}}{\rho_{p e r}} u_{\varepsilon}^{N}\right]\right]+k^{2} \mathcal{G}_{1}\left[\frac{\tilde{\mathcal{K}}_{\varepsilon}}{\mathcal{K}_{\varepsilon}} \mathcal{G}^{*}\left[\frac{\tilde{\mathcal{K}}_{\varepsilon}}{\kappa_{p e r} \mathcal{K}_{\varepsilon}} v_{\varepsilon}^{N}\right]\right] \\
-k^{2} \mathcal{G}\left[\tilde{\rho}_{\varepsilon} \mathcal{G}_{1}\left[\frac{\tilde{\mathcal{K}}_{\varepsilon}}{\mathcal{\kappa}_{\varepsilon}} v_{\varepsilon}^{N}\right]+k^{4} \mathcal{G}\left[\tilde{\rho}_{\varepsilon} \mathcal{G}\left[\tilde{\rho}_{\varepsilon} u_{\varepsilon}^{N}\right]\right]\right.
\end{aligned}
$$


which yields directly

$$
U_{\varepsilon}=k^{2} \mathcal{G}_{1}\left[\frac{\tilde{\mathcal{K}}_{\varepsilon}}{\kappa_{\varepsilon}} \mathcal{G}_{1}^{*}\left[\frac{\tilde{\rho}_{\varepsilon}}{\rho_{p e r}} u_{\varepsilon}^{N}\right]\right]+k^{2} \mathcal{G}_{1}\left[\frac{\tilde{\mathcal{K}}_{\varepsilon}}{\kappa_{\varepsilon}} \mathcal{G}^{*}\left[\frac{\tilde{\kappa}_{\varepsilon}}{\kappa_{p e r} \mathcal{K}_{\varepsilon}} v_{\varepsilon}^{N}\right]\right]-k^{2} \mathcal{G}\left[\tilde{\rho}_{\varepsilon} \mathcal{G}_{1}\left[\frac{\tilde{\mathcal{K}}_{\varepsilon}}{\mathcal{K}_{\varepsilon}} v_{\varepsilon}^{N}\right]+k^{4} \mathcal{G}\left[\tilde{\rho}_{\varepsilon} \mathcal{G}\left[\tilde{\rho}_{\varepsilon} u_{\varepsilon}^{N}\right]\right]\right.
$$

Since $u_{\varepsilon}^{N}$ is bounded on $(0, N L)$ uniformly with respect to $\omega$ and $\varepsilon$, as in the proof of Theorem 2 , the second estimate of (19) gives us

$$
\begin{aligned}
\mathbb{E}\left[\left\|\mathcal{G}_{1}\left[\frac{\tilde{\mathcal{K}}_{\varepsilon}}{\mathcal{K}_{\varepsilon}} \mathcal{G}_{1}^{*}\left[\frac{\tilde{\rho}_{\varepsilon}}{\rho_{\text {per }}} u_{\varepsilon}^{N}\right]\right]\right\|^{2}\right]+\mathbb{E}\left\|\mathcal{G}_{1}\left[\frac{\tilde{\mathcal{K}}_{\varepsilon}}{\mathcal{K}_{\varepsilon}} \mathcal{G}^{*}\left[\frac{\tilde{\mathcal{K}}_{\varepsilon}}{\kappa_{p e r} \mathcal{K}_{\varepsilon}} v_{\varepsilon}^{N}\right]\right]\right\|^{2}+\mathbb{E}\left\|\mathcal{G}\left[\tilde{\rho}_{\varepsilon} \mathcal{G}_{1}\left[\frac{\tilde{\kappa}_{\varepsilon}}{\kappa_{\varepsilon}} v_{\varepsilon}^{N}\right]\right]\right\|^{2} \\
+\mathbb{E}\left\|\mathcal{G}\left[\tilde{\rho}_{\varepsilon} \mathcal{G}\left[\tilde{\rho}_{\varepsilon} u_{\varepsilon}^{N}\right]\right]\right\|^{2} \leq C \varepsilon^{2} .
\end{aligned}
$$

This ends our proof for $u_{\varepsilon}^{N}$. The estimate for $v_{\varepsilon}^{N}$ can be demonstrated in the same manner.

Let us introduce the so-called correctors

$u_{1, \varepsilon}:=\frac{1}{\sqrt{\varepsilon}}\left(-\mathcal{G}_{1}\left[\frac{\tilde{\mathcal{K}}_{\varepsilon}}{\mathcal{K}_{\varepsilon}} v_{\text {per }}^{N}\right]+k^{2} \mathcal{G}\left[\tilde{\rho}_{\varepsilon} u_{\text {per }}^{N}\right]\right) \quad$ and $\quad v_{1, \varepsilon}:=\frac{1}{\sqrt{\varepsilon}}\left(-k^{2} \mathcal{G}_{1}^{*}\left[\frac{\tilde{\rho}_{\varepsilon}}{\rho_{\text {per }}} u_{\text {per }}^{N}\right]-k^{2} \mathcal{G}^{*}\left[\frac{\tilde{\kappa}_{\varepsilon}}{\kappa_{\text {per }} \kappa_{\mathcal{\varepsilon}}} v_{\text {per }}^{N}\right]\right)$.

and the associated DtN map

$$
\lambda_{1, \varepsilon}=v_{1, \varepsilon}(0) .
$$

These correctors depend on $\omega$, and thus would be impossible to compute, if we have only a partial knowledge on the medium, The following theorem describes however the distribution of these terms.

Theorem $3 u_{1, \varepsilon}$ and $v_{1, \varepsilon}$ converge in distribution in the space of continuous functions $\mathcal{C}([0, N L])$ to the Gaussian processes specified below

$$
\begin{aligned}
& u_{1, \varepsilon} \underset{\varepsilon \rightarrow 0}{\stackrel{\mathcal{L}}{\longrightarrow}} \int_{0}^{N L} \sigma_{u}(x, t) d W_{t}, \\
& v_{1, \varepsilon} \underset{\varepsilon \rightarrow 0}{\stackrel{\mathcal{L}}{\longrightarrow}} \int_{0}^{N L} \sigma_{v}(x, t) d W_{t} .
\end{aligned}
$$

where $W_{t}$ is a standard Brownian motion and for all $\ell \in\{u, v\}$

$$
\sigma_{\ell}(x, t)=2 \int_{0}^{\infty} \mathbb{E}\left(F_{\ell}(x, t, 0) F_{\ell}(x, t, z)\right) d z
$$

with

$$
\begin{aligned}
& F_{u}(x, t, z)=k^{2} \rho_{s}(z) G(x, t) \rho_{p e r} u_{p e r}^{N}(x)+\kappa_{s}(z) \partial_{x} G(x, t) v_{p e r}^{N}(x), \\
& F_{v}(x, t, z)=-k^{2} \rho_{s}(z) \partial_{x} G^{*}(x, t) u_{p e r}^{N}(x)+k^{2} \kappa_{s}(z) G^{*}(x, t) \frac{1}{\kappa_{p e r}} v_{p e r}^{N}(x) .
\end{aligned}
$$

Consequently, $\frac{u_{\varepsilon}^{N}-u_{p e r}^{N}}{\sqrt{\varepsilon}}$ admits the same limit in distribution in the space of square integrable functions $L^{2}((0, N L))$.

Proof We follow the result from [7] that we give here for completeness.

Theorem 4 Let $\left(Y_{n}\right)_{n \in \mathbb{N}^{*}}$ be a random process with values into $\mathcal{C}([0, D])$. $\left(Y_{n}\right)_{n \in \mathbb{N}^{*}}$ converges in law to $Y$, a random variable with values in $\mathcal{C}([0, D])$ iff

1) the finite-dimensional distribution $\left(Y_{n}\left(y_{1}\right), \cdots, Y_{n}\left(y_{p}\right)\right)$ converges to the joint distribution $\left(Y\left(y_{1}\right), \cdots, Y\left(y_{p}\right)\right)$ for any $p \in \mathbb{N}^{*}, y_{1}, \cdots, y_{p} \in[0, D]$.

2) $\left(Y_{n}\right)_{n \in \mathbb{N}^{*}}$ is tight.

The demonstration is thus accomplished in two steps. We show that $Y_{n}=u_{1, \varepsilon_{n}}$ and similarly $Z_{n}=$ $v_{1, \varepsilon_{n}}$ satisfy 1) and 2) for any $\left(\varepsilon_{n}\right)_{n}$ such that $\varepsilon \rightarrow 0$. (Step 1) First tightness is showed by proving that the following Kolmogorov criterion holds. 
Lemma 1 Let $\left(Y_{n}\right)_{n \in \mathbb{N}^{*}}$ be a random process with values into $\mathcal{C}([0, D])$. If $\left(Y_{n}\right)_{n \in \mathbb{N}^{*}}$ verifies

1) there exists $y_{0} \in[0, D], \gamma>0$ such that $\sup _{n \geq 1} \mathbb{E}\left[\left|Y_{n}\left(y_{0}\right)\right|^{\gamma}\right]<+\infty$,

and

2) there exists $\alpha, \beta>0$ such that $\forall y, y^{\prime} \in[0, D], \mathbb{E}\left[\left|Y_{n}(y)-Y_{n}\left(y^{\prime}\right)\right|^{\alpha}\right] \leq C\left|y-y^{\prime}\right|^{1+\beta}$, where $C$ does not depend on $n$,

then $\left(Y_{n}\right)_{n \in \mathbb{N}^{*}}$ is tight.

$u_{1, \varepsilon}(x)$ and $v_{1, \varepsilon}(x)$ are uniformly bounded in $\varepsilon$ in $L^{2}(\Omega)$ for any $x \in[0, N L]$. Therefore, the first assumption is verified for $\gamma=2$ and any $y_{0} \in[0, N L]$. Next we show that for all $y, y^{\prime} \in(0, N L)$ uniformly in $\varepsilon$

$$
\mathbb{E}\left[\left|u_{1, \varepsilon}(y)-u_{1, \varepsilon}\left(y^{\prime}\right)\right|^{4}\right]+\mathbb{E}\left[\left|v_{1, \varepsilon}(y)-v_{1, \varepsilon}\left(y^{\prime}\right)\right|\right]^{4} \leq C\left|y-y^{\prime}\right|^{2} .
$$

Let $y, y^{\prime} \in[0, N L] . \mathbb{E}\left[\left|u_{1, \varepsilon}(y)-u_{1, \varepsilon}\left(y^{\prime}\right)\right|^{4}\right]$ is a sum of terms of the form

$$
\frac{1}{\varepsilon^{2}} \int_{0}^{N L} \int_{0}^{N L} \int_{0}^{N L} \int_{0}^{N L} \mathbb{E}\left[a^{1}\left(t_{1}\right) a^{2}\left(t_{2}\right) a^{3}\left(t_{3}\right) a^{4}\left(t_{4}\right)\right] \prod_{i=1}^{4}\left[H^{i}\left(t_{i}, y\right)-H^{i}\left(t_{i}, y^{\prime}\right)\right] d t_{1} d t_{2} d t_{3} d t_{4},
$$

where $\left(a^{i}, H^{i}\right) \in\left\{\left\{\left(\rho_{s}, G\right),\left(\kappa_{s}, \partial_{x} G\right),\left(\rho_{s}, \bar{G}\right),\left(\kappa_{s}, \partial_{x} \bar{G}\right)\right\}\right\}$. We use once again Lemma (2.1) in [5] to bound the expectation and obtain

$$
\begin{aligned}
& \frac{1}{\varepsilon^{2}} \int_{0}^{N L} \int_{0}^{N L} \int_{0}^{N L} \int_{0}^{N L} \mathbb{E}\left(a^{1}\left(t_{1}\right) a^{2}\left(t_{2}\right) a^{3}\left(t_{3}\right) a^{4}\left(t_{4}\right)\right) \prod_{i=1}^{4}\left(H^{i}\left(t_{i}, y\right)-H^{i}\left(t_{i}, y^{\prime}\right)\right) d t_{1} d t_{2} d t_{3} d t_{4}, \\
& \leq \frac{1}{\varepsilon^{2}} \sum_{\sigma \in \Sigma} \int_{0}^{N L} \int_{0}^{N L} \int_{0}^{N L} \int_{0}^{N L} \varphi^{\frac{1}{2}}\left(\frac{\left|t_{\sigma(1)}-t_{\sigma(2)}\right|}{\varepsilon}\right) \varphi^{\frac{1}{2}}\left(\frac{\left|t_{\sigma(3)}-t_{\sigma(4)}\right|}{\varepsilon}\right) \prod_{i=1}^{4}\left|H^{i}\left(t_{i}, y\right)-H^{i}\left(t_{i}, y^{\prime}\right)\right| \\
& d t_{1} d t_{2} d t_{3} d t_{4},
\end{aligned}
$$

where $\Sigma$ is the set of permutations of $\{1, \ldots, 4\}$. This can be bounded in turn by terms of the from

$$
\left|\frac{1}{\varepsilon} \int_{0}^{N L} \int_{0}^{N L} \varphi^{\frac{1}{2}}\left(\frac{\left|s_{1}-s_{2}\right|}{\varepsilon}\right) \prod_{i=1}^{2}\right| H^{i}\left(s_{i}, y\right)-H^{i}\left(s_{i}, y^{\prime}\right)\left|d s_{1} d s_{2}\right|^{2} .
$$

If $H^{i}=G, \bar{G}$, we know directly that $\left|H^{i}\left(s_{i}, y\right)-H^{i}\left(s_{i}, y^{\prime}\right)\right| \leq C\left|y-y^{\prime}\right|$, since $G$ is Lipschitz continuous in $y$ uniformly in $s$. Therefore if at least one $H^{i}$ in the product is $G$ or $\bar{G}$, we use that $\partial_{x} G$ is bounded on $(0, N L)$ and that $\varphi^{\frac{1}{2}}|\cdot|$ is integrable to get the result for the term.

We are thus left to deal with term of the form 26 where $H^{1}, H^{2} \in\left\{\partial_{x} G, \partial_{x} \bar{G}\right\}$. In order to use that $\partial_{x} G$ is piecewise Lipschitz continuous and bounded, we divide our integration interval into 3 parts. We suppose without loss of generality that $y<y^{\prime}$. On $(0, y)$ and $\left(y^{\prime}, N L\right), \partial_{x} G$ is Lipschitz continuous uniformly in $s$ and we can bound $\left|H^{i}\left(s_{i}, y\right)-H^{i}\left(s_{i}, y^{\prime}\right)\right|$ by $C\left|y-y^{\prime}\right|$. Those terms can then be dealt as the previous ones. We are left with the term on $\left(y, y^{\prime}\right)$, on which $\partial_{x} G$ is not Lipschitz continuous. However we can use that $\partial_{x} G$ is bounded on $(0, N L)$ and that $\varphi|\cdot|$ is integrable.

$$
\begin{aligned}
\frac{1}{\varepsilon} \int_{y}^{y^{\prime}} \int_{y}^{y^{\prime}} \varphi^{\frac{1}{2}}\left(\frac{\left|s_{1}-s_{2}\right|}{\varepsilon}\right) & \left|\partial_{x} G\left(s_{1}, y\right)-\partial_{x} G\left(s_{1}, y^{\prime}\right)\right|\left|\partial_{x} G\left(s_{2}, y\right)-\partial_{x} G\left(s_{2}, y^{\prime}\right)\right| d s_{1} d s_{2} \\
& \leq C \frac{y^{\prime}-y}{\varepsilon} \int_{-\infty}^{+\infty} \varphi^{\frac{1}{2}}\left(\frac{|r|}{\varepsilon}\right) d r \leq C\left(y^{\prime}-y\right) \int_{-\infty}^{+\infty} \varphi^{\frac{1}{2}}|r| d r .
\end{aligned}
$$

This concludes the proof of the tightness.

(Step 2) Secondly, we need to prove that the finite-dimensional distributions converge to the finitedimensional distributions of the wanted limit.

Let $n \in \mathbb{N}^{*}$. Let $y_{1}, \ldots, y_{n} \in(0, N L)$. 
The finite-dimensional distribution $\left(u_{1, \varepsilon}\left(y_{1}, \omega\right), \ldots, u_{1, \varepsilon}\left(y_{1}, \omega\right)\right)$ has the following characteristic function for all $t \in \mathbb{R}^{n}$

$$
\begin{aligned}
\Phi_{\varepsilon}(t) & =\mathbb{E}\left[\prod_{j=1}^{n} e^{i t_{j} u_{1, \varepsilon}\left(y_{j}, \omega\right)}\right] \\
& =\mathbb{E}\left[\exp \left(\frac{i}{\sqrt{\varepsilon}}\left(\int_{0}^{N L} \rho_{s}\left(\frac{x}{\varepsilon}\right) f_{\rho}(x, y, t) d x+\int_{0}^{N L} \kappa_{S}\left(\frac{x}{\varepsilon}\right) f_{\mathcal{K}}(x, y, t) d x\right)\right)\right],
\end{aligned}
$$

where $f_{\rho}(x, y, t)=\sum_{j=1}^{n} t_{j} k^{2} \rho_{\text {per }}(x) G\left(x, y_{j}\right) u_{\text {per }}^{N}(x)$ and $f_{\kappa}(x, y, t)=\sum_{j=1}^{n} t_{j} \partial_{x} G\left(x, y_{j}\right) v_{\text {per }}^{N}$.

The demonstration relies therefore on the convergence in distribution of oscillatory integrals of the type $\frac{1}{\sqrt{\varepsilon}} \int_{0}^{L} f\left(\frac{t}{\varepsilon}\right) m(t) d t$ where $m$ is a continuous function and $f$ a stationary $\rho$-mixing process with an integrable coefficient. Those integrals have been thoroughly studied, see for example [21,16, 5] and are proved to converge in distribution to $\int_{0}^{D} m(x) \sigma d W_{x}$, where $\sigma^{2}=2 \int_{0}^{\infty} R_{f}(r) d r$. In particuler, in Theorem (2.8) of [5], one can find a clear idea of the demonstration of this result.

The convergence of $\frac{u_{\varepsilon}^{N}-u_{\text {per }}^{N}}{\sqrt{\varepsilon}}$ comes consequently from the estimate in Proposition 5

\subsection{Numerical simulations}

We present in this section the results of the numerical simulations that we conducted in the homogenization regime. Our goal is to illustrate and verify the theoretical results that we proved above and to show how our approach can be used to build approximated transparent boundary conditions.

\subsubsection{Randomly perturbed periodic media in the homogenization regime}

Our first step is to construct numerically the two zero-mean jointly stationary strongly mixing processes $v_{s}^{\kappa}$ and $v_{s}^{\rho}$. Following [3], to obtain a stationary finite-range dependent or strongly mixing process, a scalar white noise $W$, i.e. a sequence of independent standard normal random variables, is generated and convolved with a bump function $f$. The desired process is then a smooth function of this convolution. We choose in our simulations to take the sin of the convolution to preserve the zero-mean property. The width and decreasing rate of the bump function gives you the correlation length of the generated process and the strongly mixing coefficient $\varphi$. On Figure 1, we plotted an example of a scalar white noise, a bump function $f: x \rightarrow e^{-\frac{1}{1-x^{2}}} \mathbb{1}_{|x|<1}$ and the zero-mean stationary strongly mixing process obtained through the sin of the convolution of the two former. Note that with this specific example of bump function, the process has a finite range of dependence of 2 . The two processes $v_{s}^{\kappa}$ and $v_{s}^{\rho}$ are generated in the same way but from different white noises. This gives us two independent processes that therefore verify the jointly mixing and stationary hypothesis.

Equipped with the two processes $v_{s}^{\kappa}$ and $v_{s}^{\rho}$, we can now simulate periodic media perturbed by oscillating stationary processes. We choose a 1-periodic background medium characterized by the two parameters

$$
\kappa_{\text {per }}^{-1}=1.1+\sin (2 \pi \cdot) \text { and } \rho_{\text {per }}=1.1+\cos (2 \pi \cdot),
$$

that we perturb to obtain $\kappa_{\varepsilon}^{-1}=\kappa_{\text {per }}^{-1}\left(1+v_{s}^{\kappa}(\dot{\bar{\epsilon}})\right)$ and $\rho_{\varepsilon}=\rho_{\text {per }}\left(1+v_{s}^{\rho}(\dot{\bar{\epsilon}})\right)$. We represented on Figure 2 two realisations of the randomly perturbed periodic coefficient $\rho_{\varepsilon}$ for two different $\varepsilon$. On the left in green $\varepsilon=0.01$ and on the left in blue $\varepsilon=0.2$. As $\varepsilon$ goes to 0 , the medium oscillates faster and faster. This oscillatory behavior hints at the difficulties of computing exact solutions in this type of media. Indeed, the faster the oscillation, the smaller the needed space discretization.

Different realizations of the medium lead to different solutions of 9 ). To give you an idea of the variety of solutions that we deal with, we plotted on Figure 3 three different solutions of (9) associated to three different media generated as described above for two different $\varepsilon$. On the left, $\varepsilon=0.05$ and on the right, $\varepsilon=0.2$. 


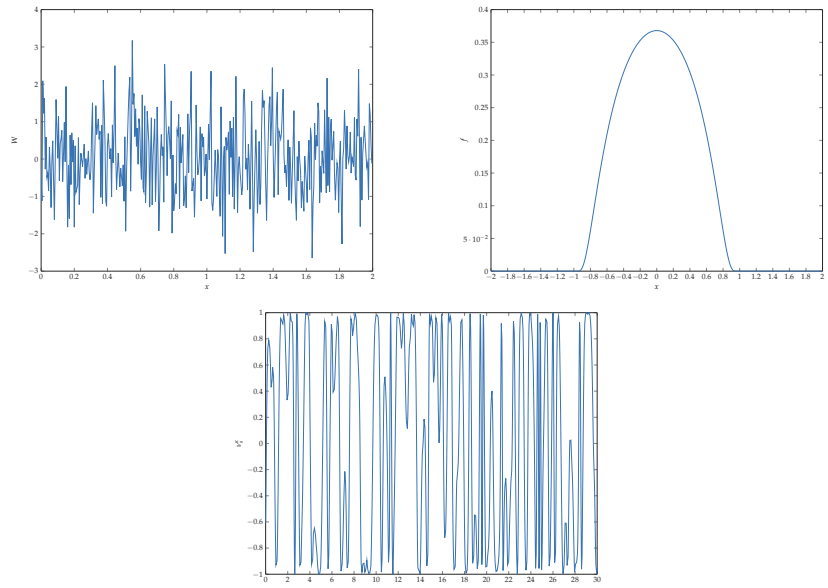

Fig. 1: White noise $W$, bump function $f$ and zero-mean stationary strongly mixing process $v_{s}^{k}$ obtained by convolution of $W$ and $f$
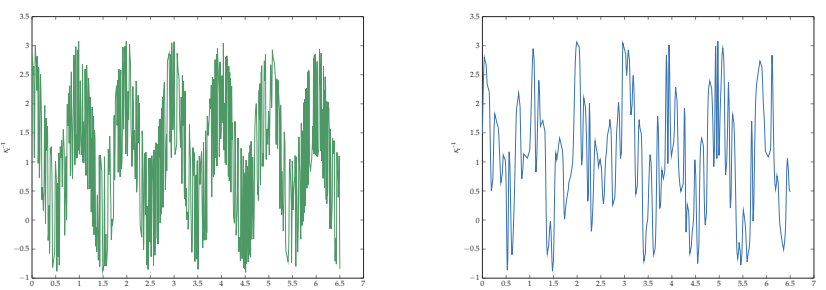

Fig. 2: $\rho_{\varepsilon}$ with respect to $x$ for two different $\omega$ and two different $\varepsilon: \varepsilon=0.01$ (left) and $\varepsilon=0.2$ (right)
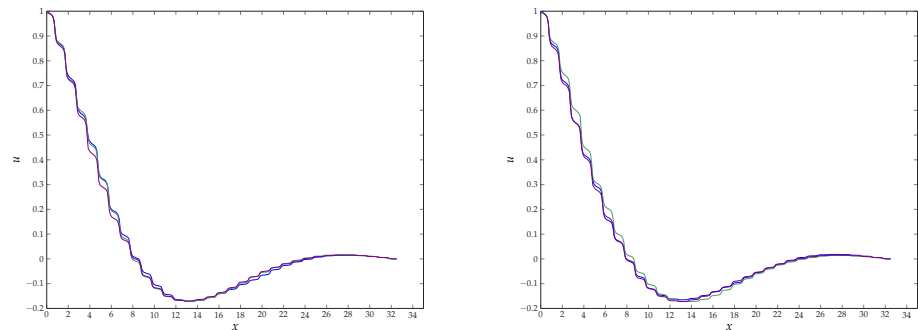

Fig. 3: $u_{\varepsilon}^{N}$ for different $\omega$ and $\varepsilon=0.05$ (left) and $\varepsilon=0.2$ (right).

\subsubsection{Asymptotic behavior of $u_{\varepsilon}^{N}$}

The first result that we want to corroborate is the convergence result of Therorem 2 We choose a frequency of 0.1 and a damping coefficient $\eta=0.2$. Before solving the equation, we compute thanks to Proposition 3 the number of periods $N$, that we need to consider our problem on, in order to obtain a good-enough approximation of our solution on the half-line, say for example an error of $10^{-5}$. We then solve the equation on $(0, N L)$ for 300 different randomly generated media over a range of $\varepsilon$ from 0.05 to 0.2. By the Monte-Carlo method, we deduce, for each $\varepsilon$, the norm in $L^{2}\left(\Omega, L^{2}((0, N L))\right)$ of $u_{\varepsilon}^{N}-u_{p e r}^{N}$ and the norm in $L^{2}(\Omega)$ of the associated DtN coefficient error $\lambda_{\varepsilon}^{N}-\lambda_{\text {per }}^{N}$. In Figure 4 are represented in blue $\log \left\|u_{\varepsilon}^{N}-u_{p e r}^{N}\right\|_{L^{2}\left(\Omega, L^{2}((0, N L))\right)}$ and in green $\left.\log \left\|\lambda_{\varepsilon}^{N}-\lambda_{p e r}^{N}\right\|_{L^{2}(\Omega}\right)$ with respect to $\log (\varepsilon)$. We recover the theoretical rate of convergence of $\sqrt{\varepsilon}$ in the slope of 0.5 .

Next, we want to exhibit the convergence in law of the corrector stated in Theorem 3 . We display this result for the DtN coefficient but note that we could have printed the exact same plots for the corrector of $u_{\varepsilon}^{N}$ or the flux $v_{\varepsilon}^{N}$ at any point $x \in(0, N L)$. We represented on Figure 7, on the left, the 


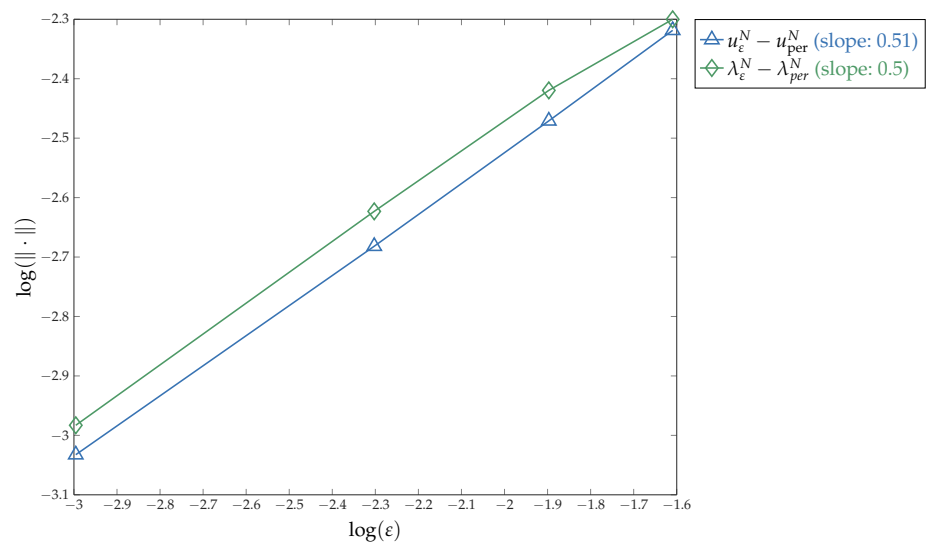

Fig. 4: Rate of convergence of $u_{\varepsilon}^{N}$ in $L^{2}\left(\Omega, L^{2}((0, N L))\right)$ and $\lambda_{\varepsilon}^{N}$ in $L^{2}(\Omega)$ to resp. $u_{p e r}^{N}$ and $\lambda_{p e r}^{N}$.

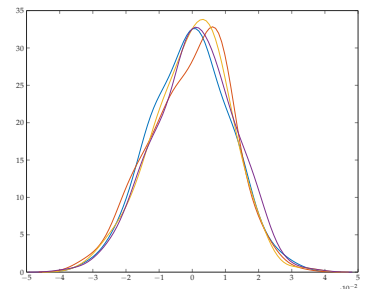

(a)

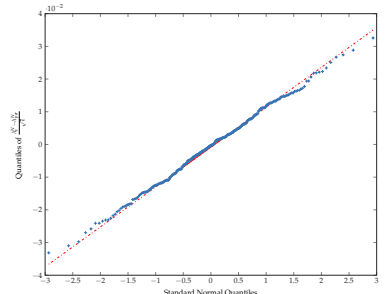

(b)

Fig. 5: (a) Probability density of $\frac{\lambda_{\varepsilon}^{N}-\lambda_{\text {per }}^{N}}{\sqrt{\varepsilon}}$ as $\varepsilon$ goes to 0 , (b) Normal quantile-quantile plot of the corrector $\frac{\lambda_{\varepsilon}^{N}-\lambda_{\text {per }}^{N}}{\sqrt{\varepsilon}}$ for $\varepsilon=0.05$.

probability density of $\lambda_{1, \varepsilon}=\frac{\lambda_{\varepsilon}^{N}-\lambda_{p e r}^{N}}{\sqrt{\varepsilon}}$, for different $\varepsilon$ ranging from 0.05 to 0.2 , approximated over 300 simulations per $\varepsilon$. One can see that, as $\varepsilon$ goes to 0 , the probability density of $\lambda_{1, \varepsilon}$ converges to the density of a centered normal random variable. To quantify more accurately this limit behavior, we plotted on the right the normal quantile-quantile plot of the same quantity. On the $x$-axis are represented the quantiles of a normal random variable and on the $y$-axis the quantiles of our data. If the corrector follows as $\varepsilon$ goes to 0 a normal distribution, its quantiles should accumulate on the red line. We can see that it is here the case for $\varepsilon=0.05$.

\subsubsection{Transparent boundary conditions}

In this last section, we expose how our approach can be of use in the construction of transparent boundary conditions for randomly perturbed periodic media. We want to solve the wave equation on the half-line $[-1,+\infty]$, where $[-1,0]$ is a known homogeneous medium with $\kappa_{\text {hom }}^{-1}=\rho_{\text {hom }}=1$ and $[0,+\infty)$ is a randomly perturbed periodic medium modeled as above. $u_{\varepsilon}$ is thus the unique solution in $H^{1}((-1,+\infty))$ of

$$
\left\{\begin{array}{l}
-\partial_{x} \kappa_{\varepsilon} \partial_{x} u_{\varepsilon}-k^{2} \rho_{\varepsilon} u_{\varepsilon}=0 \quad \text { in }(-1, N L), \\
u_{\varepsilon}(-1)=1, \quad u_{\varepsilon}(N L)=0 .
\end{array}\right.
$$

We know that the above problem is equivalent as solving

$$
\left\{\begin{array}{l}
-\partial_{x} \kappa_{h o m} \partial_{x} u_{\varepsilon}-\rho_{h o m} k^{2} u_{\varepsilon}=0 \\
u_{\varepsilon}(-1)=1, \quad \partial_{x} u_{\varepsilon}(0)=\lambda_{\varepsilon}^{+} u_{\varepsilon}(0),
\end{array} \quad \text { in }(-1,0),\right.
$$



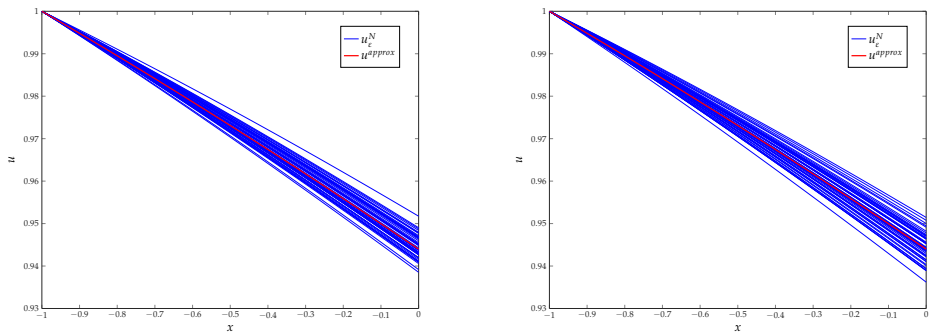

Fig. 6: Solution $u$ in $[-1,0]$ with exact and approximated DtN conditions for $\varepsilon=0.05$ (left) and $\varepsilon=0.1$ (right).

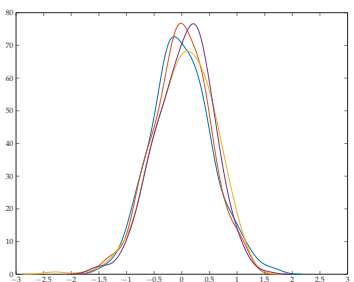

(a)

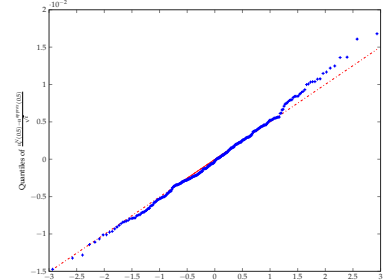

(b)

Fig. 7: (a) Probability density of $\frac{u_{\varepsilon}^{N}(-0.5)-u^{a p p r o x}(-0.5)}{\sqrt{\varepsilon}}$ as $\varepsilon$ goes to 0 , (b) Normal quantile-quantile plot of the corrector $\frac{u_{\varepsilon}^{N}(-0.5)-u^{a p p r o x}(-0.5)}{\sqrt{\varepsilon}}$ for $\varepsilon=0.05$.

where $\lambda_{\varepsilon}^{+}$is the DtN coefficient associated to the problem of $[0,+\infty)$. We approximate $\lambda_{\varepsilon}^{+}$by $\lambda_{\text {per }}^{N}$ and construct an approximation of $u_{\varepsilon}$ on $[-1,0]$ that is cheap to compute. We solve on $[0,1]$ the homogeneous problem

$$
\left\{\begin{array}{c}
-\partial_{x} \kappa_{\text {hom }} \partial_{x} u^{\text {approx }}-\rho_{\text {hom }} k^{2} u^{\text {approx }}=0 \quad \text { in }(-1,0), \\
u^{\text {approx }}(-1)=1, \quad \partial_{x} u^{\text {approx }}(0)=\lambda_{\text {per }}^{N} u^{\text {approx }}(0) .
\end{array}\right.
$$

On Figure 6 are represented in blue different solutions $u_{\varepsilon}^{N}$ corresponding to exact DtN boundary conditions associated to different random media occupying $(0, N L)$ and in red the approximated solution $u^{a p p r o x}$ corresponding to the approximated DtN boundary condition. On the left, $\varepsilon=0.05$ and on the right, $\varepsilon=0.1$. We can not predict the almost sure behavior of $u_{\varepsilon}^{N}$. However, one can notice that as $\varepsilon$ goes to 0 the width of the different realizations of $u_{\varepsilon}^{N}$ diminishes.

As proven in Theorem 3, we know nonetheless the limit distribution of the corrector $u_{1, \varepsilon}:=$ $\frac{u_{\varepsilon}^{N}-u^{a p p r o x}}{\sqrt{\varepsilon}}$ as $\varepsilon$ goes to 0 . To verify this result, we plotted in Figure 7 on the left, the probability density of $u_{1, \varepsilon}(-0.5)$ for different $\varepsilon$ ranging from 0.05 to 0.2 approximated over 300 simulations per $\varepsilon$. The distribution of $u_{1, \varepsilon}$ seems to converge to a Gaussian as $\varepsilon$ goes to 0 . This is confirmed by the normal quantile-quantile plot of the same quantity on the right for $\varepsilon=0.05$. The quantiles of the data samples are well aligned on the red line representing the quantile of a normal law. We have thus access to an approximation of order $\sqrt{\varepsilon}$ in law of the exact solution $u_{\varepsilon}$. This gives us among others all its statistics and its cumulative distribution function at an order of $\sqrt{\varepsilon}$ for small $\varepsilon$. 


\section{Rare perturbations case}

In this section, let $\left(B_{\varepsilon}^{j}\right)_{j \in \mathbb{N}}$ be independent Bernouilli random variables with parameter $\varepsilon \in[0,1]$, we consider a random medium occupying $\mathbb{R}^{+}$, characterized by the following coefficients

$$
\begin{array}{ll}
\text { for a.e. }(x, \omega) \in \mathbb{R}^{+} \times \Omega, & \kappa_{\varepsilon}(x, \omega)=\kappa_{\mathrm{per}}(x)+b_{\varepsilon}(x, \omega) \tilde{\kappa}_{\mathrm{per}}(x), \\
& \rho_{\varepsilon}(x, \omega)=\rho_{\mathrm{per}}(x)+b_{\varepsilon}(x, \omega) \tilde{\rho}_{\mathrm{per}}(x),
\end{array}
$$

where $\kappa_{\text {per, }}, \tilde{\kappa}_{\text {per }}, \rho_{\text {per }}, \tilde{\rho}_{\text {per }}$ are $L$-periodic functions with $L>0$ and the random perturbation $b_{\varepsilon}$ is given by

$$
b_{\varepsilon}(x, \omega)=\sum_{j \in \mathbb{N}} \mathbb{1}_{I_{j}}(x) B_{\varepsilon}^{j}(\omega), \quad I_{j}=[j L,(j+1) L] .
$$

We recall that we investigate for this case the asymptotic expansion of the solution $u_{\varepsilon}^{N}$ of 9 in terms of $\varepsilon$ and deduce an expansion for the associated DtN coefficient defined in (11).

\subsection{The limit behaviour}

As the probability $\varepsilon$ for each period to be altered goes to 0 , it is natural to think that the solution $u_{\varepsilon}^{N}$ converges, in a sense to be determined, to the solution $u_{\mathrm{per}}^{N}$ of the problem 41] with the periodic coefficients $\left(\kappa_{\text {per }}, \rho_{\text {per }}\right)$. We prove in this section that indeed $u_{\varepsilon}^{N}$ converges to $u_{\text {per }}^{N}$ as $\varepsilon \rightarrow 0$ in $L^{2}\left(\Omega, H^{1}((0, N L))\right)$ but only at a rate of $\sqrt{\varepsilon}$ as in the homogenization case. The weak convergence rate in $L^{2}(\Omega \times(0, N L))$ is slightly better.

Theorem 5 (i) There exists a constant $C>0$ such that

$$
\left\|u_{\varepsilon}^{N}-u_{p e r}^{N}\right\|_{L^{2}\left(\Omega, H^{1}((0, N L))\right.} \leq C \sqrt{\varepsilon}\left\|u_{p e r}^{N}\right\|_{H^{1}((0, N L))},
$$

and

$$
\left|\lambda_{\varepsilon}^{N}-\lambda_{\text {per }}^{N}\right| \leq C \sqrt{\varepsilon}
$$

where $C=\max \left(\kappa_{+}, \rho_{+}|k|^{2}\right)\left[\Im(k)|k| \min \left(\frac{\kappa_{-}}{|k|^{2}}, \rho_{-}\right)\right]^{-1}$.

(ii) Weakly in $L^{2}(\Omega \times(0, N L)), u_{\varepsilon}^{N}$ admits the 0 -order asymptotic expansion

$$
u_{\varepsilon}^{N}=u_{p e r}^{N}+o(\sqrt{\varepsilon})
$$

Proof (i) Let $r_{\varepsilon}^{0}=u_{\varepsilon}^{N}-u_{\text {per }}^{N}$. It is easy to see that $r_{\varepsilon}^{0}$ is the unique solution in $L^{2}\left(\Omega, H^{1}((0, N L))\right.$ of

$$
\left\{\begin{array}{l}
-\partial_{x}\left[\kappa_{\varepsilon} \partial_{x} r_{\varepsilon}^{0}\right]-k^{2} \rho_{\varepsilon} r_{\varepsilon}^{0}=\partial_{x}\left[b_{\varepsilon} \tilde{\kappa}_{\mathrm{per}} \partial_{x} u_{\mathrm{per}}^{N}\right]+b_{\varepsilon} \tilde{\rho}_{\mathrm{per}} k^{2} u_{\mathrm{per}}^{N}, \quad \text { in }(0, N L), \\
r_{\varepsilon}^{0}(0)=0, \quad r_{\varepsilon}^{0}(N L)=0 .
\end{array}\right.
$$

Using Proposition 2, we can show that

$$
\left\|r_{\varepsilon}^{0}\right\|_{L^{2}\left(\Omega, H^{1}((0, N L))\right)} \leq C\left(\left\|b_{\varepsilon} \partial_{x} u_{\mathrm{per}}^{N}\right\|_{L^{2}\left(\Omega, L^{2}((0, N L))\right)}+\left\|b_{\mathcal{\varepsilon}} u_{\mathrm{per}}^{N}\right\|_{L^{2}\left(\Omega, L^{2}((0, N L))\right)}\right)
$$

where $C=\max \left(\kappa_{+}, \rho_{+}|k|^{2}\right)\left[\Im(k)|k| \min \left(\frac{\kappa_{-}}{|k|^{2}}, \rho_{-}\right)\right]^{-1}$.

We can compute similarly each term appearing in the right hand side of the previous inequality. For instance,

$$
\begin{aligned}
\left\|b_{\varepsilon} u_{\mathrm{per}}\right\|_{L^{2}\left(\Omega, L^{2}((0, N L))\right.}^{2} & =\mathbb{E} \sum_{j=0}^{N-1} \int_{j L}^{(j+1) L}\left(B_{\varepsilon}^{j}(\omega)\right)^{2}\left|u_{\mathrm{per}}^{N}(x)\right|^{2} d x \\
& =\sum_{j=0}^{N-1} \mathbb{E}\left[B_{\varepsilon}^{j}(\omega)\right] \int_{j L}^{(j+1) L}\left|u_{\mathrm{per}}^{N}(x)\right|^{2} d x=\varepsilon\left\|u_{\mathrm{per}}^{N}\right\|_{L^{2}((0, N L))}^{2}
\end{aligned}
$$


which, combined with (32), enables to obtain (42).

(ii) Let $w_{\varepsilon}^{0}:=\frac{r_{\varepsilon}^{0}}{\sqrt{\varepsilon}} \cdot w_{\varepsilon}^{0}$ is bounded in $L^{2}\left(\Omega, H^{1}((0, N L))\right)$ thanks to (i). Thus $w_{\varepsilon}^{0}$ and $\partial w_{\varepsilon}^{0}$ are bounded in $L^{2}(\Omega \times(0, N L))$. As $\kappa_{\varepsilon}$ is uniformly bounded, we know that $\kappa_{\varepsilon} \partial_{x} w^{0} \varepsilon$ is also bounded in $L^{2}(\Omega \times$ $(0, N L))$. Up to extraction, $w_{\varepsilon}^{0}$ and $\kappa_{\varepsilon} \partial_{x} w^{0} \varepsilon$ converge weakly in $L^{2}(\Omega \times(0, N L))$. Moreover, since $\left\|b_{\varepsilon}\right\|_{L^{2}(\Omega \times(0, N L))}=\sqrt{\varepsilon N L}, b_{\varepsilon} / \sqrt{\varepsilon}$ converges weakly in $L^{2}(\Omega \times(0, N L))$, up to extraction, to a limit $b_{0}$. Since it converges to 0 in probability, we know that $b_{0}=0$. We divide 31 by $\sqrt{\varepsilon}$, take the limit of the variational formulation as $\varepsilon$ to 0 and obtain that $r_{\varepsilon}^{0}=o(\sqrt{\varepsilon})$.

\subsection{First-order corrections}

We want to refine the approximation obtained in the previous section. We know that at a fixed period $I_{j}=(j L,(j+1) L)$, the periodic coefficients are perturbed with a probability $\varepsilon$. A natural intuition is that a more accurate approximation of $u_{\varepsilon}$ could be obtained by considering the solutions of the problem where the periodic coefficients are perturbed in one and only one period. Let us then introduce for all $j \in\{0, \ldots, N-1\}, u_{j}^{N}$ the unique solution in $H^{1}((0, N L))$ of

$$
\left\{\begin{array}{lr}
-\partial_{x}\left[\left(\kappa_{\mathrm{per}}+\mathbb{1}_{I_{j}} \tilde{\kappa}_{\mathrm{per}}\right) \partial_{x} u_{j}^{N}\right]-k^{2}\left(\rho_{\mathrm{per}}+\mathbb{1}_{I_{j_{m}}} \tilde{\rho}_{\mathrm{per}}\right) u_{j}^{N}=0 \\
u_{j}^{N}(0)=1, \quad u_{j}^{N}(N L)=0 . & \text { in }(0, N L),
\end{array}\right.
$$

and $\lambda_{j}^{N}$ the associated DtN coefficient

$$
\lambda_{j}^{N}:=-\left[\left(\kappa_{\text {per }}+\mathbb{1}_{I_{j}}\right) \partial_{x} u_{j}^{N}\right](0) .
$$

More generally, let $p$ be an integer in $\{1, \ldots, N\}$ and $j_{1}<\ldots<j_{p}$ be $p$ indices in $\{0, \ldots, N-1\}$, we consider the periodic medium where exactly only the periods $I_{j_{1}}, \ldots, I_{j_{p}}$ are perturbed. This medium is characterized by the coefficients

$$
\kappa_{\text {per }}+\sum_{m=1}^{p} \mathbb{1}_{I_{j m}} \tilde{\kappa}_{\text {per }} \quad \text { and } \quad \rho_{\text {per }}+\sum_{m=1}^{p} \mathbb{1}_{I_{m}} \tilde{\rho}_{\text {per }}
$$

We denote $u_{j_{1}, \ldots, j_{p}}^{N}$ the unique solution in $H^{1}((0, N L))$ of

$$
\left\{\begin{array}{lr}
-\partial_{x}\left[\left(\kappa_{\text {per }}+\sum_{m=1}^{p} \mathbb{1}_{I_{j_{m}}} \tilde{\kappa}_{\text {per }}\right) \partial_{x} u_{j_{1}, \ldots, j_{p}}^{N}\right]-k^{2}\left(\rho_{\text {per }}+\sum_{m=1}^{p} \mathbb{1}_{I_{j_{m}}} \tilde{\rho}_{\text {per }}\right) u_{j_{1}, \ldots, j_{p}}^{N}=0 \\
u_{j_{1}, \ldots, j_{p}}^{N}(0)=1, \quad u_{j_{1}, \ldots, j_{p}}^{N}(N L)=0 . & \text { in }(0, N L),
\end{array}\right.
$$

and $\lambda_{j}^{N}$ the associated DtN coefficient

$$
\lambda_{j_{1}, \ldots, j_{p}}^{N}:=-\left[\left(\kappa_{\text {per }}+\sum_{m=1}^{p} \mathbb{1}_{I_{j_{m}}} \tilde{\kappa}_{\text {per }}\right) \partial_{x} u_{j_{1}, \ldots, j_{p}}^{N}\right](0) .
$$

For a.e. $\omega \in \Omega$, we denote $\mathcal{P}_{\omega}$ the set defined by

$$
\mathcal{P}_{\omega}:=\left\{j \in\{0, \ldots, N-1\}, B_{\varepsilon}^{j}(\omega)=1\right\} .
$$

It is easy to see that $u_{\varepsilon}^{N}$ can be written for a.e. $(x, \omega) \in(0, N L) \times \Omega$

$$
u_{\varepsilon}^{N}(x, \omega)=\mathbb{1}_{\mathcal{P}_{\omega}=\varnothing}(\omega) u_{\mathrm{per}}^{N}(x)+\sum_{p=1}^{N-1} \sum_{j_{1}<\ldots<j_{p}} \mathbb{1}_{\mathcal{P}_{\omega}=\left\{j_{1}, \ldots, j_{p}\right\}}(\omega) u_{j_{1}, \ldots, j_{p}}^{N}(x) .
$$


The expectation of $u_{\varepsilon}^{N}$ has therefore the following expression

$$
\mathbb{E}\left(u_{\varepsilon}^{N}\right)=(1-\varepsilon)^{N} u_{\text {per }}+\varepsilon^{p}(1-\varepsilon)^{N-p} \sum_{p=1}^{N} \sum_{j_{1}<\ldots<j p} u_{j_{1}, \ldots, j_{p}}^{N}
$$

which yields directly the following result.

Theorem 6 In mean, $u_{\varepsilon}$ and $\lambda_{\varepsilon}$ verify the following first-order asymptotic expansions

$$
\begin{gathered}
\left\|\mathbb{E}\left[u_{\varepsilon}^{N}\right]-u_{\text {per }}^{N}-\varepsilon \sum_{j=0}^{N-1}\left(u_{j}^{N}-u_{p e r}^{N}\right)\right\|_{H^{1}((0, N L))}=\mathcal{O}\left(\varepsilon^{2}\right), \\
\left|\mathbb{E}\left[\lambda_{\varepsilon}^{N}\right]-\lambda_{p e r}^{N}-\varepsilon \sum_{j=0}^{N-1}\left(\lambda_{j}^{N}-\lambda_{p e r}^{N}\right)\right|=\mathcal{O}\left(\varepsilon^{2}\right) .
\end{gathered}
$$

Remark 2 Higher order approximations can also be derived from (36). For example, the second order corrector reads

$$
\left\|\mathbb{E}\left[u_{\varepsilon}^{N}\right]-u_{p e r}^{N}-\varepsilon \sum_{j=0}^{N-1}\left(u_{j}^{N}-u_{p e r}^{N}\right)-\varepsilon^{2} \sum_{i \neq j} u_{i, j}^{N}-2 u_{i}^{N}+u_{p e r}^{N}\right\|_{H^{1}((0, N L))}=\mathcal{O}\left(\varepsilon^{2}\right) .
$$

However, from a numerical point of view, we did not feel the need of taking into accounts higher order approximations.

The first-order correction for $\mathbb{E}\left(u_{\varepsilon}^{N}\right)$ is indeed linked to the solutions in a medium with one and only one one defect. The advantage of such expansion is that each term is solution of a deterministic problem set on a locally perturbed periodic medium. Using [15], these problems can be solved considering only cell problems with $\left(\kappa_{\text {per }}, \rho_{\text {per }}\right)$ or $\left(\kappa_{\text {per }}+\tilde{\kappa}_{\text {per }}, \rho_{\text {per }}+\tilde{\rho}_{\text {per }}\right)$. Computationally, the resolution is then really cheap.

Unfortunately, adding this corrector to $u_{\varepsilon}^{N}$ does not change the rate of convergence found in Theorem 4.4 in $L^{2}\left(\Omega, H^{1}((0, N L))\right.$, as stated in the following proposition. Figure 10 illustrates the different rates of convergence that we exhibited so far.

Proposition 6 There exists a constant $C$ such that

$$
\left\|u_{\varepsilon}^{N}-u_{p e r}^{N}-\varepsilon \sum_{j=0}^{N-1}\left(u_{j}^{N}-u_{p e r}^{N}\right)\right\|_{L^{2}\left(\Omega, H^{1}((0, N L))\right)} \leq C \sqrt{\varepsilon}
$$

and

$$
\left|\lambda_{\varepsilon}^{N}-\lambda_{\text {per }}^{N}-\varepsilon \sum_{j=0}^{N-1}\left(\lambda_{j}^{N}-\lambda_{p e r}^{N}\right)\right| \leq C \sqrt{\varepsilon}
$$

Proof For all $j \in\{0, \ldots, N-1\}$, let consider $v_{j}^{N}=u_{j}^{N}-u_{\mathrm{per}}^{N}$. It is the unique solution in $H^{1}((0, N L))$ of

$$
\left\{\begin{aligned}
-\partial_{x} & {\left[\left(\kappa_{\mathrm{per}}+\mathbb{1}_{I_{j}} \tilde{\kappa}_{\mathrm{per}}\right)\right] \partial_{x} v_{j}^{N}-k^{2}\left(\rho_{\mathrm{per}}+\mathbb{1}_{I_{j}} \tilde{\rho}_{\mathrm{per}}\right) v_{j}^{N} } \\
& =\partial_{x}\left[\mathbb{1}_{I_{j}} \tilde{\kappa}_{\mathrm{per}} \partial_{x} u_{\mathrm{per}}^{N}\right]+k^{2} \mathbb{1}_{I_{j}} \tilde{\rho}_{\mathrm{per}} u_{\mathrm{per}}^{N}, \quad \text { in }(0, N L), \\
v_{j}^{N}(0) & =0, \quad v_{j}^{N}(N L)=0 .
\end{aligned}\right.
$$

For all $j \in\{0, \ldots, N-1\}$, we have the estimate

$$
\left\|v_{j}^{N}\right\|_{H^{1}((0, N L))} \leq C\left\|u_{\mathrm{per}}^{N}\right\|_{H^{1}((j L,(j+1) L))}
$$


for a positive constant $C$.

Let us introduce $r_{\varepsilon}^{1}=u_{\varepsilon}^{N}-u_{\mathrm{per}}^{N}-\varepsilon \sum_{j=0}^{N-1} v_{j}^{N}$. It is the unique solution in $L^{2}\left(\Omega, H^{2}((0, N L))\right)$ of

$$
\left\{\begin{aligned}
-\partial_{x}\left[\kappa_{\varepsilon} \partial_{x} r_{\varepsilon}^{1}\right]-k^{2} \rho_{\varepsilon} r_{\varepsilon}^{1} & =\partial_{x}\left[\tilde{\mathcal{K}}_{\text {per }}\left(b_{\varepsilon}-\varepsilon\right) \partial_{x} u_{\text {per }}^{N}\right]+k^{2} \tilde{\rho}_{\text {per }}\left(b_{\varepsilon}-\varepsilon\right) u_{\text {per }}^{N} \\
& +\varepsilon\left[\partial_{x}\left[\tilde{\mathcal{K}}_{\text {per }} b_{\varepsilon} \partial_{x} \sum_{j=0}^{N-1} v_{j}^{N}\right]+k^{2} \tilde{\rho}_{\text {per }} b_{\varepsilon} \sum_{j=0}^{N-1} v_{j}^{N}\right] \\
& -\varepsilon\left[\partial_{x}\left[\tilde{\kappa}_{\text {per }} \sum_{j=0}^{N-1} \mathbb{1}_{I_{j}} \partial_{x} v_{j}^{N}\right]+k^{2} \tilde{\rho}_{\text {per }} \sum_{j=0}^{N-1} \mathbb{1}_{I_{j}} v_{j}^{N}\right], \\
& \text { in }(0, N L), \\
r_{\varepsilon}^{1}(0)=0, \quad r_{\varepsilon}^{1}(N L)=0 . &
\end{aligned}\right.
$$

As in the proof of Theorem 4.4 and using Proposition 2 and we have that

$$
\begin{array}{r}
\left\|r_{\varepsilon}^{1}\right\|_{L^{2}\left(\Omega, H^{1}((0, N L))\right.} \leq C\left[\left\|\left(b_{\varepsilon}-\varepsilon\right) \partial_{x} u_{\mathrm{per}}^{N}\right\|_{L^{2}\left(\Omega, L^{2}((0, N L))\right)}+\left\|\left(b_{\varepsilon}-\varepsilon\right) u_{\mathrm{per}}^{N}\right\|_{L^{2}\left(\Omega, L^{2}((0, N L))\right)}\right. \\
\left.+\varepsilon\left[\left\|b_{\varepsilon} \partial_{x} v_{j}^{N}\right\|_{L^{2}\left(\Omega, L^{2}((0, N L))\right.}+\left\|b_{\varepsilon} v_{j}^{N}\right\|_{L^{2}\left(\Omega, L^{2}((0, N L))\right.}\right]+\varepsilon\left\|\sum_{j=0}^{N-1} \mathbb{1}_{I_{j}} v_{j}^{N}\right\|_{H^{1}}\right],
\end{array}
$$

and then

$$
\left\|r_{\varepsilon}^{1}\right\|_{L^{2}\left(\Omega, H^{1}((0, N L))\right.} \leq C\left[\sqrt{\varepsilon(1-\varepsilon)}\left\|u_{\mathrm{per}}^{N}\right\|_{H^{1}}+\varepsilon^{3 / 2}\left\|\sum_{j=0}^{N-1} v_{j}^{N}\right\|_{H^{1}}+\varepsilon\left\|\sum_{j=0}^{N-1} \mathbb{1}_{I_{j}} v_{j}^{N}\right\|_{H^{1}}\right] .
$$

However, similarly as (36), one can obtain from (35) asymptotic expansions for the expectation of any smooth function of $u_{\varepsilon}^{N}$. Higher order approximations can also be derived.

Theorem 7 For any $\varphi \in \mathcal{C}_{c}^{\infty}(\mathbb{R})$

$$
\begin{gathered}
\left\|\mathbb{E}\left[\varphi\left(u_{\varepsilon}^{N}\right)\right]-\varphi\left(u_{p e r}^{N}\right)-\varepsilon \sum_{j=0}^{N-1} \varphi\left(u_{j}^{N}\right)-\varphi\left(u_{p e r}^{N}\right)\right\|_{H^{1}((0, N L))}=\mathcal{O}\left(\varepsilon^{2}\right), \\
\left.\mid \mathbb{E}\left[\varphi\left(u_{\varepsilon}^{N}\right)\right)\right]-\varphi\left(\lambda_{p e r}^{N}\right)-\varepsilon \sum_{j=0}^{N-1} \varphi\left(\lambda_{j}^{N}\right)-\varphi\left(\lambda_{p e r}^{N}\right) \mid=\mathcal{O}\left(\varepsilon^{2}\right),
\end{gathered}
$$

This can be seen as an approximation in law in the first order in $\varepsilon$ of $u_{\varepsilon}^{N}$. Indeed, let $P_{u_{\varepsilon}^{N}}$ be the distribution of $u_{\varepsilon}^{N}$. Let $\mathcal{P}(\mathbb{R})$ be the set of probability measures with compact support on $\mathbb{R}$. $\operatorname{In} \mathcal{P}(\mathbb{R})$, the following asymptotic expansion holds weakly-*

$$
P_{\lambda_{\varepsilon}^{N}}=\delta_{u_{\mathrm{per}}^{N}}+\varepsilon \sum_{j=0}^{N-1} \delta_{u_{j}^{N}}-\delta_{u_{\mathrm{per}}^{N}}+\mathcal{O}\left(\varepsilon^{2}\right) .
$$

\subsection{Numerical simulations}

We present, in this section, a few simulation results to illustrate our theorems and exhibit how our approach can provide a tool to build transparent boundary conditions for the studied randomly perturbed periodic media.

We start off by exposing in Figure 8 two random realizations of the perturbed periodic media that we consider for two different $\varepsilon$. We take our two parameters $\kappa_{p e r}^{-1}$ and $\rho_{\text {per }}$ of the periodic medium to be

$$
\cos (2 \pi \cdot)+2,
$$



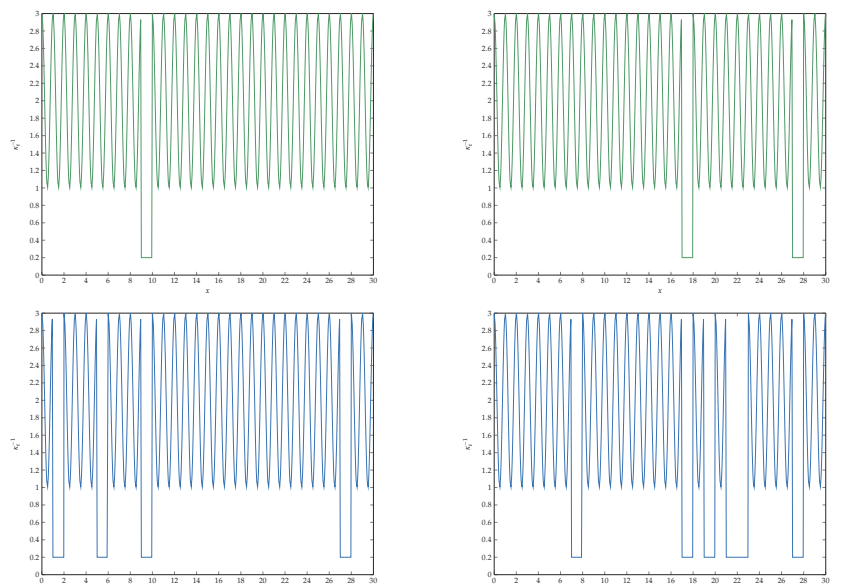

Fig. 8: $\kappa_{\varepsilon}^{-1}$ with respect to $x$ for two different $\omega$ and two different $\eta: \varepsilon=0.05$ (top) and $\varepsilon=0.2$ (bottom)
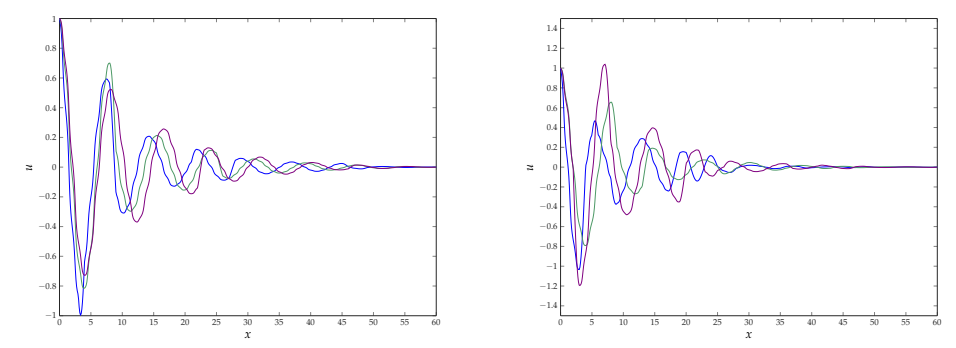

Fig. 9: $u_{\varepsilon}^{N}$ for different $\omega$ and $\varepsilon=0.05$ (left) and $\varepsilon=0.2$ (right).

and perturb each period with a probability $\varepsilon$ by $\tilde{\kappa}_{\text {per }}$ and $\tilde{\rho}_{\text {per }}$ such that

$$
\kappa_{p e r}+\tilde{\kappa}_{p e r}=\rho_{\text {per }}+\tilde{\rho}_{\text {per }}=0.2 .
$$

The parameters of the medium stay this way bounded above and below. The top row represents two different random realizations of $\kappa_{\varepsilon}^{-1}$ for $\varepsilon=0.05$, that is a medium where each period has a probability of $1 / 20$ to be altered, and the bottom row represents two different random realizations of $\kappa_{\varepsilon}^{-1}$ for $\varepsilon=0.2$, that is a medium where each period has a probability of $1 / 5$ to be altered.

For the two same $\varepsilon$, we plot in Figure 9 three different solutions $u_{\varepsilon}^{N}$ obtained by solving the equation in three random media generated as described above. We chose a frequency of 1 and a damping coefficient of 0.2. Before solving the system, we compute, using Proposition 3 , the appropriate number $N$ of periods to write our equation on, such that the error between the truncated problem and the problem on the positive half-line is as small as desired, for example $10^{-6}$.

\subsubsection{Different modes of convergence and their orders}

The first results that we verify numerically are the rates of convergence proved in the two previous sections. We chose 8 values of $\varepsilon$ between 0.05 and 0.4 and solved the equations for 2000 different realizations of the medium for each value of $\varepsilon$. Note that we need more simulations than in the homogenization regime since we are working here with rare perturbations.

We computed by the Monte-Carlo method $\mathbb{E}\left[u_{\varepsilon}^{N}\right]$ and plotted the log of the norm of the error in $H^{1}((0, N L))$ of the $0-, 1$ st- and 2nd- order asymptotic expansion, with respect to the log of $\varepsilon$. One can see in Figure 10 that the green lines with triangle markers have a slope of respectively 0.8, 1.7 and 2.5, for the $0-, 1$ st- and 2nd- order approximations, close to what we expected from Theorem 6 i.e. resp. 1,2 and 3 . 


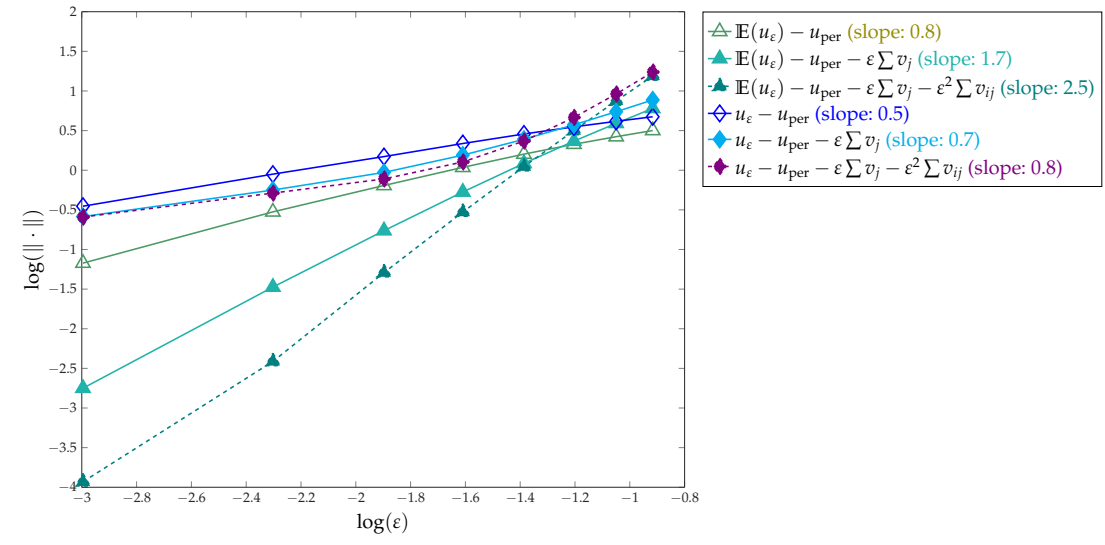

Fig. 10: Rates of convergence of $\mathbb{E}\left(u_{\varepsilon}^{N}\right)$ and $u_{\varepsilon}^{N}$ and their first-order corrections

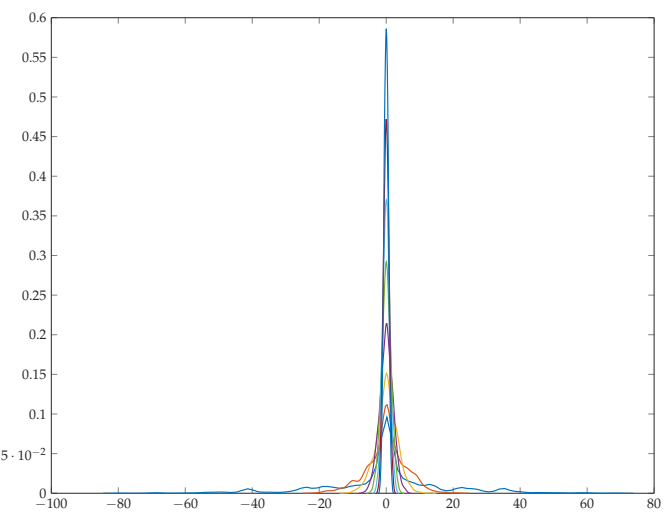

Fig. 11: Probability density of $\varepsilon^{-2}\left(\lambda_{\varepsilon}-\lambda_{\text {per }}-\varepsilon \sum \lambda_{j}-\lambda_{\text {per }}\right)$ as $\varepsilon$ goes to 0

Similarly, as Theorem 4.4. and Proposition 6 predicted, when we plot the log of the error in $L^{2}\left(\Omega, H^{1}((0, N L))\right)$ of the 0 -, 1 st- and 2nd- order asymptotic expansion of $u_{\varepsilon}^{N}$, with respect to the $\log$ of $\varepsilon$, we find that the slopes do not change and stay around 0.5. On Figure 10 , those lines correspond to the blue, cyan and magenta lines with diamond markers. We find slopes of $0.5,0.7$ and 0.8 respectively. Once again, to compute the expectation that appear in the norm, we used the MonteCarlo method.

Note that we computed for this figure the 2nd order approximation. Here $v_{i, j}=u_{i, j}^{N}-2 u_{i}^{N}+u_{p e r}$ for all $i \neq j \in[|1, N|]$. Recall that $u_{i, j}^{N}$ denotes the solution of the equation in a medium where only the $i$ th and $j$ th periods are perturbed.

Finally, we want to illustrate the last convergence result stated Theorem 7 , i.e. the convergence in law of the 1st order asymptotic expansion on $u_{\varepsilon}^{N}$ with a rate of $\varepsilon^{2}$. Since a similar result holds for the Dirichlet-to-Neumann coefficient, we chose to represent this result on it. We thus computed the DtN coefficients associated to the solutions in the 2000 realizations of our medium over the range of $\varepsilon$. We approximated over this sample the probability density of $\varepsilon^{-2}\left(\lambda_{\varepsilon}-\lambda_{\text {per }}-\varepsilon \sum \lambda_{j}-\lambda_{\text {per }}\right)$ for each $\epsilon$. We then plotted those density functions in Figure 11. You can see that as $\epsilon$ goes to 0 the density function converges to a dirac as expected.

\subsubsection{Homogenization regime}

A second verification that we pursued is to compare our results with those of A. Anantharaman and C. Le Bris. They obtain in [2] an asymptotic expansion for the homogenized coefficient of the 

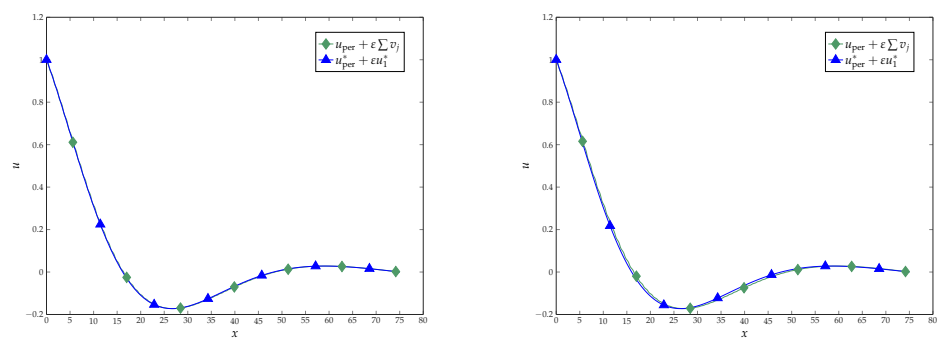

Fig. 12: Comparison between our asymptotic expansion and [A. Anantharaman and C. Le Bris]'s expansion for $\varepsilon=0.05$ (left) and $\varepsilon=0.2$ (right).

elliptic pde in the same media that we studied but in any dimension $d \geq 1$ and of course in the homogenization regime. For $d=1$, they derive an exact expression for the first order expression of the homogenized coefficient $a_{\varepsilon}$. We used this expression to compute an approximation of the solution $u_{\varepsilon}^{N}$ in the low frequency regime (frequency of 0.1) and compared it to our first order approximation. In Figures 12, we represented in green with diamond markers our approximation and in blue with triangle markers their approximations for two different $\varepsilon, \varepsilon=0.05$ on the left and $\varepsilon=0.2$ on the right. One can see that they match in both cases.

\subsubsection{Transparent conditions and DtN}

We consider a homogeneous medium on $[-1,0]$, where $\kappa_{\text {hom }}=\rho_{\text {hom }}=1$, and we want to solve the wave equation with a Dirichlet condition at -1

$$
\left\{\begin{array}{l}
-\partial_{x} \kappa_{\varepsilon} \partial_{x} u_{\varepsilon}-k^{2} \rho_{\varepsilon} u_{\varepsilon}=0 \quad \text { in }(-1, N L), \\
u_{\varepsilon}(-1)=1, \quad u_{\varepsilon}(N L)=0
\end{array}\right.
$$

The randomly perturbed periodic medium lies in $(0,+\infty)$ as before. As explained in the introduction, the restriction of this solution to $(-1,0)$ is the unique solution of the following equation with a boundary condition of the form 4 at 0

$$
\left\{\begin{array}{l}
-\partial_{x} \kappa_{h o m} \partial_{x} u_{\varepsilon}-\rho_{h o m} k^{2} u_{\varepsilon}=0 \quad \text { in }(-1,0), \\
u_{\varepsilon}(-1)=1, \quad \partial_{x} u_{\varepsilon}(0)=\lambda_{\varepsilon}^{+} u_{\varepsilon}(0)
\end{array}\right.
$$

We use Theorem 7 to approximate at a first order in law $\lambda_{\varepsilon}$ and construct an approximation of $u_{\varepsilon}$ on $[-1,0]$ that is cheap to compute. We solve

$$
\left\{\begin{array}{l}
-\partial_{x} \kappa_{h o m} \partial_{x} u_{\varepsilon}^{\text {approx }}-\rho_{\text {hom }} k^{2} u_{\varepsilon}^{\text {approx }}=0 \text { in }(-1,0) \\
u_{\varepsilon}^{\text {approx }}(-1)=1, \quad \partial_{x} u_{\varepsilon}^{\text {approx }}(0)=\left(\lambda_{\text {per }}^{N}+\epsilon \sum_{j=0}^{N-1} \lambda_{j}^{N}-\lambda_{p e r}^{N}\right) u_{\varepsilon}^{\text {approx }}(0)
\end{array}\right.
$$

which is equivalent to imposing $u_{\varepsilon}^{a p p r o x}=u_{p e r}^{N}+\varepsilon \sum_{j=0}^{N-1} v_{j}^{N}$ on $(0, N L) \cdot u_{\varepsilon}^{a p p r o x}$ provides therefore a first order approximation in law of $u_{\varepsilon}$ on our bounded know domain $(-1,0)$ bordering the unknown random medium.

We plotted in Figure 13 a few random realisations of the true solution $u_{\varepsilon}$ on $[-1,0]$ for two different $\varepsilon$, on the left $\varepsilon=0.05$ and on the right $\varepsilon=0.1$. We represented as well the two approximated solutions obtained by taking the 0 order (in green) and 1st order (in red) approximation of the DtN coefficient in our boundary condition. Here the frequency is high and taken to be 20. Even though our approximated solutions seem to provide a good estimate for $u_{\varepsilon}$, it seems that the 1st order approximation does not make at first sight a significant difference in bettering the estimate compared to the 0 order. This is not surprising however, because we know that the convergence of $u_{\varepsilon}^{\text {approx }}$ to $u_{\varepsilon}$ does not hold almost surely but in law. 

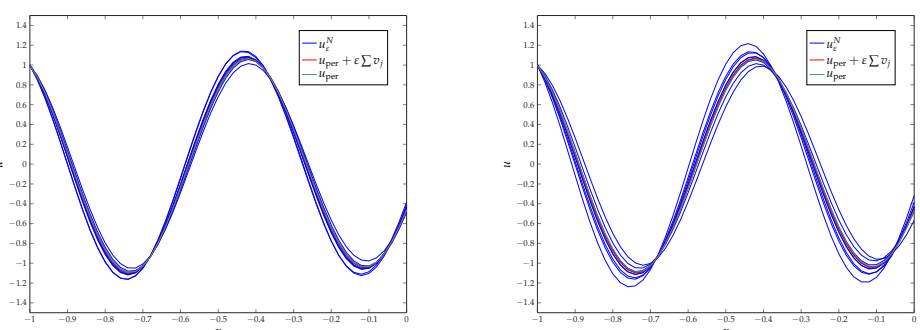

Fig. 13: Solution $u$ in $[-1,0]$ with exact and approximated DtN conditions for $\varepsilon=0.05$ (left) and $\varepsilon=0.1$ (right).

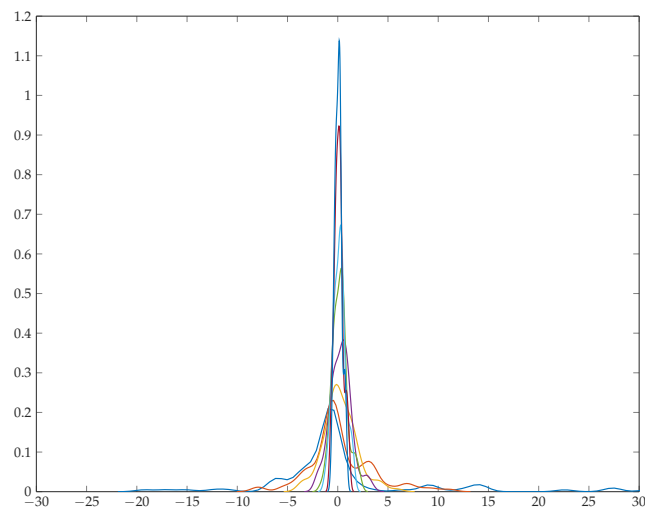

Fig. 14: Probability density of $\varepsilon^{-2}\left(u_{\varepsilon}(-0.5)-u_{\varepsilon}^{\text {approx }}(-0.5)\right)$ as $\varepsilon$ goes to 0

We thus exhibited the convergence in law of $u_{\varepsilon}^{\text {approx }}$ to $u_{\varepsilon}$ by representing the probability density of $\varepsilon^{-2}\left(u_{\varepsilon}(-0.5)-u_{\varepsilon}^{\text {approx }}(-0.5)\right)$ in Figure 14 for different $\varepsilon$. Like before, the probability density is computed over 2000 simulations per $\varepsilon$. A similar figure could be constructed for each point $x \in$ $[-1,0]$. This result might be a justification for using the 1 st order approximation against the 0 -order since the convergence in law to a dirac is faster for the former.

\subsection{Other randomly perturbed periodic media}

We consider a more general setting, where the random term $b_{\varepsilon}$ in the perturbation follows on each period, independently of the others, a distribution $\mathbb{P}_{B_{\varepsilon}}$, which is not a Bernouilli distribution anymore but any distribution that is rare in the sense that it verifies the properties enounced below. We express thus $b_{\varepsilon}$ as follows

$$
\forall(x, \omega) \in \mathbb{R}_{+}^{*} \times \Omega, \quad \tilde{b}_{\varepsilon}(x, \omega)=\sum_{j \in \mathbb{N}} \mathbb{1}_{I_{j}}(x) \tilde{B}_{\varepsilon}^{j}(\omega),
$$

where $\left(\tilde{B}_{\varepsilon}^{j}\right)_{j \in \mathbb{N}}$ are i.i.d. random variables in $L^{2}(\Omega)$. We suppose that $\tilde{B}_{\varepsilon}$ is uniformly bounded in $\omega$ and $\varepsilon$ by a constant $K<M$ and that its second moment $\mathbb{E}\left(\left(\tilde{B}_{\varepsilon}\right)^{2}\right)$ converges to 0 as $\varepsilon \rightarrow 0$. M is chosen such that

$$
\kappa_{\text {per }} \pm M \tilde{\kappa}_{\text {per }} \text { and } \rho_{\text {per }} \pm M \tilde{\rho}_{\text {per }}
$$

still verify 1 This allows us to write the equivalent of theorem in this setting

Theorem $8 u_{\varepsilon}^{N}$ converges to $u_{0}^{N}$ at the following converging rate

$$
\left\|u_{\varepsilon}^{N}-u_{p e r}^{N}\right\|_{L^{2}\left(\Omega, H^{1}((0, N L))\right)} \leq C\left\|\tilde{B}_{\varepsilon}\right\|_{L^{2}(\Omega)}\left\|u_{p e r}^{N}\right\|_{H^{1}((0, N L))} .
$$


To obtain the first order asymptotic expansion in law, we need to impose an asymptotic assumption on $P_{\tilde{B}_{\varepsilon}}$. Note that the Bernouilli law of parameter $\varepsilon$ verifies

$$
P_{B_{\varepsilon}}=\delta_{0}+\varepsilon\left(\delta_{1}-\delta_{0}\right)+\mathcal{O}\left(\varepsilon^{2}\right), \quad \text { weakly- }{ }^{*} \text { in } \mathcal{P}((0,1))
$$

We follow then the first section and assume that

$$
P_{\tilde{B}_{\varepsilon}}=\delta_{0}+\varepsilon P^{1}+\mathcal{O}\left(\varepsilon^{2}\right), \quad \text { weakly-* in } \mathcal{P}((-M, M)),
$$

i.e. for all $\varphi \in \mathcal{C}_{c}^{\infty}((-M, M))$

$$
\left|\mathbb{E}\left(\varphi\left(\tilde{B}_{\varepsilon}\right)\right)-\varphi(0)-\varepsilon \int \varphi d P^{1}\right|=\mathcal{O}\left(\varepsilon^{2}\right) .
$$

In order to exhibit the first order term in our asymptotic expansion, we introduce $u_{s_{0}, \cdots, s_{N-1}}^{N}$, for $s_{1}, \cdots, s_{N} \in(-M, M)$, the solution of the equation in a medium where the $j$ th period is perturbed at a level $s_{j}$, i.e. $b_{\mathcal{\varepsilon}}^{j}(\omega)=s_{j}$ for $j \in[|1, n|]$.

$u_{s_{0}, \cdots, s_{N-1}}^{N}$ is the solution in $H^{1}((0, N L))$ of the following equation

$$
\left\{\begin{array}{lr}
-\partial_{x}\left(\kappa_{\text {per }}+\sum_{j=0}^{N-1} s_{j} \mathbb{1}_{I_{j}} \tilde{\kappa}_{\text {per }}\right) \partial_{x} u_{s_{0}, \cdots, s_{N-1}}^{N}-k^{2}\left(\rho_{\text {per }}+\sum_{j=0}^{N-1} s_{j} \mathbb{1}_{I_{j}} \tilde{\rho}_{\text {per }}\right) u_{s_{0}, \cdots, s_{N-1}}^{N}=0 \\
u_{s_{0}, \cdots, s_{N-1}}^{N}(0)=1, \quad u_{s_{0}, \cdots, s_{N-1}}^{N}(N L)=0 . & \text { in }(0, N L),
\end{array}\right.
$$

We can show by induction that

$$
\begin{array}{rlr}
F:(-M, M)^{N} & \rightarrow & H^{1}((0, N L)) \\
\left(s_{0}, \cdots s_{N-1}\right) & \mapsto & u_{s_{0}, \cdots, s_{N-1}}^{N}
\end{array}
$$

is in $\left.\mathcal{C}^{\infty}\left((-M, M)^{N}\right)\right)$.

We can then express $\mathbb{E}\left(\varphi\left(u_{\mathcal{\varepsilon}}^{N}\right)\right)$ for any $\varphi \in \mathcal{C}_{\mathcal{c}}(\mathbb{R})$

$$
\forall \varphi \in \mathcal{C}_{c}(\mathbb{R}), \mathbb{E}\left(\varphi\left(u_{\varepsilon}^{N}\right)\right)=\int_{(-M, M)^{N}} \varphi\left(u_{s_{0}, \cdots, s_{N-1}}^{N}\right) \prod_{j=0}^{N-1} P_{B_{\varepsilon}}\left(d s_{j}\right) .
$$

We use our asymptotic expansion on $P_{B_{\varepsilon}}$ to obtain the expansion for the product. Weakly-* in $\mathcal{P}(-M, M)$

$$
\prod_{j=0}^{N-1} P_{b_{\varepsilon}}\left(d s_{j}\right)=\prod_{j=0}^{N-1} \delta_{0}\left(d s_{j}\right)+\varepsilon \sum_{j=0}^{N-1} P^{1}\left(d s_{j}\right) \prod_{l \neq j} \delta_{0}\left(d s_{l}\right)+\mathcal{O}\left(\varepsilon^{2}\right)
$$

We plug this expansion into 43 and obtain the following theorem.

Theorem 9 In $H^{1}((0, N L))$, the following expansion holds for all $\varphi \in \mathcal{C}_{c}(\mathbb{R})$

$$
\mathbb{E}\left(\varphi\left(u_{\varepsilon}^{N}\right)\right)=\varphi\left(u_{p e r}^{N}\right)+\varepsilon \sum_{j=0}^{N-1} \int_{-M}^{M} \varphi\left(u_{0, \cdots, s_{j}, \cdots, 0}^{N}\right) P^{1}\left(d s_{j}\right)+\mathcal{O}\left(\varepsilon^{2}\right),
$$

i.e. weakly-* in $\mathcal{P}(\mathbb{R}), P_{u_{\varepsilon}^{N}}$ verifies

$$
P_{u_{\varepsilon}^{N}}=\delta_{u_{p e r}^{N}}+\varepsilon \sum_{j=0}^{N-1} \int_{-M}^{M} \delta_{u_{0, \cdots, s_{j}, \cdots, 0}} P^{1}\left(d s_{j}\right)+\mathcal{O}\left(\varepsilon^{2}\right)
$$




\section{Conclusion}

We have proposed different approximations of the DtN coefficient for two types of weakly randomly perturbed periodic media. This analysis is for now based on the exponential decay of the solution in presence of dissipation. Indeed this allows us to approximate the solution by the solution of a problem set on a truncated domain. We think that this analysis could be extended in absence of dissipation when the frequency lies in the so-called spectral gaps of the underlying periodic operator. This will be the subject of a forthcoming paper. One extension that we are also looking at, is to consider other weakly random perturbations of periodic media where the wavelength is still large with respect to the correlation length of the random processes but is small compared to the distance propagation of the wave.

\section{References}

1. Anantharaman, A. and C. Le Bris, Elements of mathematical foundations for a numerical approach for weakly random homogenization problems, Communications in Computational Physics, Global Science Press, 11 (4) (2012), pp. 1103-1143.

2. Anantharaman, A. and C. Le Bris, A numerical approach related to defect-type theories for some weakly random problems in homogenization, Multiscale Modeling and Simulation, SIAM, 9 (2) (2011), pp. 513-544.

3. Armstrong, S., T. Kuusi and J.C. Mourrat, The additive structure of elliptic homogenization. Inventiones mathematicae, 208(3), 999-1154, 2017.

4. Ash, M. , W. Kohler, G. Papanicolaou, M. Postel and B. White, Frequency content of randomly scattered signals, SIAM Rev. 33, 519-625 (1991).

5. Bal, G. , Central limits and homogenization in random media, Multiscale modeling $\mathcal{E}$ Simulation, 7 (2008) 677-702.

6. Bella, P., B. Fehrman, J. Fischer and F. Otto, Stochastic homogenization of linear elliptic equations: Higher-order error estimates in weak norms via second-order correctors. SIAM Journal on Mathematical Analysis, 49(6) (2017), pp. 4658-4703.

7. Billingsley P., Convergence of Probability Measures, John Wiley and Sons, New York, (1999).

8. Bolthausen, E. , On the central limit theorem for stationary mixing random fields, Ann. Probab., 10 (1982), pp. 1047-1050.

9. Feng, X., J. Lin, and C. Lorton, An Efficient Numerical Method for Acoustic Wave Scattering in Random Media, SIAM/ASA J. uncertainty quantification, 3 (2015), pp. 790-822.

10. Figari, R., E. Orlandi, and G. Papanicolaou, Mean field and Gaussian approximation for partial differential equations with random coefficients, SIAM J. Appl. Math., 42 (1982), pp.1069-1077.

11. Fliss, S., Etude mathématique et numérique de la propagation des ondes dans des milieux périodiques localement perturbés. Diss. PhD thesis, Ecole Polytechnique, 2009.

12. Fouque, J.-P., J. Garnier, G.P. Papanicolaou, and K. Sølna, Wave Propagation and Time Reversal in Randomly Layered Media, Springer (2007).

13. Garnier, J., Wave propagation in one-dimensional random media, Panoramas et Synthéses, 12, pp. 101-138, (2001).

14. Gloria, A. and Otto, F., The corrector in stochastic homogenization: optimal rates, stochastic integrability, and fluctuations, arXiv preprint arXiv:1510.08290 (2015).

15. Joly, P. J.R. Li and S. Fliss, Exact boundary conditions for periodic waveguides containing a local perturbation, Communications in Computational Physics, 1 (6), 945-973, 2006.

16. Khas'minskii, R. On stochastic processes defined by differential equations with a small parameter, Theory Probab. Appl., 11 (1966), pp. 211-228.

17. Klyaskin, V. I., Stochastic equations and waves in random media, Nauka, Moscow, (1980).

18. Kozlov, S.M., Averaging of random operators, Matematicheskii Sbornik, 151(2), 188-202, 1979.

19. Krengel, U., Ergodic Theorems, de Gruyter Studies in Mathematics, 6 (1985), de Gruyter.

20. Mourrat, J-C., First-order expansion of homogenized coefficients under Bernoulli perturbations, J. Math. Pures Appl., 103 (2015), pp. 68-101.

21. Papanicolaou, G. and W. Kohler, Asymptotic theory of mixing stochastic ordinary differential equations, Comm. Pure Appl. Math., 27 (1974), pp. 641-668.

22. Papanicolaou, G.C. and S. R. S. Varadhan, Boundary value problems with rapidly oscillating random coefficients, Random fields, I, II (Esztergom, 1979), Colloquia mathematica Societatis Jànos Bolyai, 27 (1981), pp. 835-873.

23. Pembery, O. R. and E. A. Spence, The Helmholtz equation in random media: well-posedness and a priori bounds, arXiv:1805.00282.

24. Shiryaev, A.N., Probability, Graduate Texts in Mathematics, 95 (1984), Springer. 